\title{
The Fate of Unstable Gauge Flux Compactifications
}

\author{
C.P. Burgess ${ }^{a, 41}$, S.L. Parameswarand2 and I. Zavalad 3 \\ ${ }^{a}$ Department of Physics and Astronomy, McMaster University, \\ 1280 Main Street West, Hamilton ON, L8S 4M1, Canada. \\ ${ }^{b}$ Perimeter Institute for Theoretical Physics, \\ 31 Caroline Street North, Waterloo ON, N2L 2Y, Canada. \\ ${ }^{c}$ II. Institut für Theoretische Physik der Universität Hamburg, \\ DESY Theory Group, Notkestrasse 85, Bldg. 2a, D-22603 Hamburg, Germany. \\ ${ }^{d}$ Bethe Center for Theoretical Physics and \\ Physikalisches Institut der Universität Bonn, \\ Nußallee 12, D-53115 Bonn, Germany.
}

\begin{abstract}
Fluxes are widely used to stabilise extra dimensions, but the supporting monopolelike configurations are often unstable, particularly if they arise as gauge flux within a non-abelian gauge sector. We here seek the endpoint geometries to which this instability leads, focussing on the simplest concrete examples: sphere-monopole compactifications in six dimensions. Without gravity most monopoles in non-abelian gauge groups are unstable, decaying into the unique stable monopole in the same topological class. We show that the same is true in Einstein-YM systems, with the new twist that the decay leads to a shrinkage in the size of the extra dimensions and curves the non-compact directions: in $D$ dimensions a $\operatorname{Mink}_{D-2} \times S_{2}$ geometry supported by an unstable monopole relaxes to $\mathrm{AdS}_{D-2} \times S_{2}$, with the endpoint sphere smaller than the initial one. For supergravity the situation is more complicated because the dilaton obstructs such a simple evolution. The endpoint instead acquires a dilaton gradient, thereby breaking some of the spacetime symmetries. For $6 \mathrm{D}$ supergravity we argue that it is the 4D symmetries that break, and examine several candidates for the endpoint geometry. By using the trick of dimensional oxidation it is possible to recast the supergravity system as a higher-dimensional Einstein-YM monopole, allowing understanding of this system to guide us to the corresponding endpoint. The result is a Kasner-like geometry conformal to $\mathrm{Mink}_{4} \times S_{2}$, with nontrivial conformal factor and dilaton breaking the maximal $4 \mathrm{D}$ symmetry and generating a singularity. Yet the resulting configuration has a lower potential energy than did the initial one, and is perturbatively stable, making it a sensible candidate endpoint for the evolution.
\end{abstract}

\footnotetext{
${ }^{1}$ Email: cburgess@perimeterinstitute.ca

${ }^{2}$ Email: susha.louise.parameswaran@desy.de

${ }^{3}$ Email: zavala@th.physik.uni-bonn.de
} 


\section{Contents}

1 Introduction $\quad 2$

2 Theory and Background 4

2.1 Background solutions . . . . . . . . . . . . . . . . . 5

2.2 Linearised instability . . . . . . . . . . . . . . . . . . 8

2.3 Topology ............................ . . . . . . . . . . . .

3 The Instability's Endpoint 12

3.1 Einstein Yang-Mills Theory ... . . . . . . . . . . . 13

4 Endpoint Revisited: Including the Dilaton 16

$4.14 \mathrm{D}$ or 2D: Which symmetries break? . . . . . . . . . . . . . . 17

4.2 Supersymmetric $\mathrm{AdS}_{3} \times \tilde{S}_{3} \ldots \ldots \ldots \ldots \ldots$

4.3 Deking the Dilaton . . . . . . . . . . . . . . . . . . 19

4.3.1 Oxidation/Reduction . . . . . . . . . . . . . . 20

4.3.2 The Rugby Ball and its Decay . . . . . . . . . . . . . . . . 21

4.3.3 The Decay of Warped Configurations . . . . . . . . . . . . 25

5 Conclusions 31

\section{Introduction}

The ubiquity of moduli in extra-dimensional compactifications has been a persistent thorn in the side of model-builders attempting to bring higher-dimensional theories into contact with Nature as we see it around us. For this reason flux-supported compactifications, for which various $n$-form field strengths thread cycles and are topologically blocked from relaxing to zero, represent a significant step forward by providing an attractive mechanism that dynamically stabilises many of these moduli.

Better yet, the required $n$-form fields are as common as dirt in supersymmetric theories, arising as components of the gravity supermultiplet in higher dimensions; as Maxwell fields required by anomaly cancellation; or as fields sourced by D-branes or other such objects. Perhaps the simplest such construction, due to Salam and Sezgin [1], is more than 20 years old, and threads a Maxwell flux through the extra dimensions in 6D supergravity to stabilise its compactification to $\mathrm{Mink}_{4} \times S_{2}$.

What is less well known is that a great many of such monopole configurations are unstable, particularly when the flux involved arises as a Dirac monopole embedded into a non-Abelian gauge sector. For instance, explicit calculations [2] show that sphere-monopole compactifications in anomaly-free supergravity - and their warped braneworld generalizations - are generically unstable, even though the monopole in question carries nontrivial topological charge. The instability is possible because there are typically more monopole solutions than there are distinct topological sectors, allowing most to decay to the (often unique) stable representative in any topological class - a phenomenon that is well understood within pure Yang Mills (YM) theories [3, 4, 5]. 
For monopole-supported systems, the coupling to gravity does not remove the instability [6, 7], and requires the geometry also to relax as the monopole decays. We examine this relaxation here, and argue that it is fairly straightforward for the Einstein-YM system (EYM). As in pure YM theory, the unstable monopole evolves towards the unique stable monopole in the same topological class [5], and as it does so the geometry adjusts simply by shrinking the size of the supported extra-dimensional sphere, and by curving the large 4 dimensions. In $d+2$ dimensions, starting from $\operatorname{Mink}_{d} \times S_{2}$ the system evolves towards $\mathrm{AdS}_{d} \times S_{2}^{\prime}$, with the radius of $S_{2}^{\prime}$ being smaller than that of $S_{2}$.

The situation is more complicated in the supergravity case, because the dilaton obstructs this same simple evolution towards another maximally symmetric solution built with the stable monopole because for it $\square \sigma \neq 0$. As a result $\partial_{M} \sigma \neq 0$, instead leading to a breakdown of some of the spacetime symmetries. The corresponding final state for higher dimensional systems, with gravity and a dilaton back-reacting to the monopole dynamics, is unknown.

In this paper we examine several candidate stable endpoint configurations for the simplest case of compactifications of $6 \mathrm{D}$ supergravity down to $4 \mathrm{D}$. We argue that the insensitivity of the low-energy effective 4D scalar potential to scalar gradients in the compactified two dimensions make it likely that it is the $4 \mathrm{D}$ spacetime symmetries that break in this case, rather than those of the compactified two dimensions.

To find the endpoint solution we employ a trick: a cycle of dimensional oxidation and reduction that maps the solutions of the supergravity of interest onto those of a dilatonfree pure EYM system in still-higher dimensions. We use this to map the unstable initial monopole-supported supergravity configuration onto an unstable monopole-supported state in the still-higher dimensional theory. Assuming this higher-dimensional EYM system relaxes in the simple maximally-symmetric way tells us its endpoint, and this can then be mapped to determine the endpoint EYM-dilaton configuration that is supported by the final stable state into which the monopole decays.

Proceeding in this way we are led to a stable, nonsupersymmetric endpoint geometry that (in the absence of brane sources) is conformal to $\mathrm{Mink}_{4} \times S_{2}$. Its nontrivial conformal factor and dilaton break the maximal 4D symmetry, giving rise to a singular geometry for which the dilaton and curvature blow up at a point in the $4 \mathrm{D}$ spacetime. However, the configuration nonetheless has a lower potential energy than did the initial one, and is stable, and is a reasonable candidate for the endpoint of the instability.

Although the dilaton changes the dynamics drastically, the presence of branes (specifically, conical singularities in the extra-dimensional geometry) and warping do not make much difference, as we show by also finding a warped generalization of the endpoint solution in this case, for generic brane tensions. The solutions we find in this way turn out to be static analogues of the time-dependent scaling solutions to 6D supergravity found in [8], with the fields varying along a $4 \mathrm{D}$ spatial coordinate rather than along time.

We also examine a class of supersymmetric solutions to $6 \mathrm{D}$ supergravity as candidate endpoints (that also break the 4D spacetime symmetry) [9]. Although we cannot prove these not to be the ultimate endpoint, we provide arguments as to why this seems less likely than those we construct using the oxidation/reduction trick.

The rest of our exposition is organised as follows. The next section, $\S 1$, summarises the field equations of chiral gauged 6D supergravity [13], together with their most general monopole-supported solutions that have at most conical singularities [14, 15]. This section 
concludes by briefly summarising the linearised stability analysis of ref. [2], and reviewing the topological classification of non-abelian Dirac monopoles in YM theories. $\S 3$ then describes how gravity backreacts to monopole decay in dilaton-free EYM theory, by shrinking the extra dimensions and curving the 4 large dimensions. Finally $\S 4$ generalises these considerations to the EYM-dilaton system that arises in the 6D supergravity of interest. This section describes the oxidation/reduction procedure, and applies it to two examples. The first example considers unwarped systems such as arise in the absence of branes, or with two branes having equal tension. The second does the case of the general warped geometries of $\S 2$, having only conical singularities. We end with some brief conclusions.

\section{Theory and Background}

We start with the bosonic action 4 for chiral 6D gauged supergravity coupled to gauge- and hyper-multiplet matter, with gauge group $\hat{\mathcal{G}}=\mathcal{G} \times U(1)_{R}[13]$

$$
\begin{aligned}
S_{B}=\int d^{6} x \sqrt{-g}[ & \frac{1}{\kappa^{2}} R-\frac{1}{4} \partial_{M} \sigma \partial^{M} \sigma-\frac{1}{4} e^{\kappa \sigma / 2} \operatorname{Tr}\left(F_{M N} F^{M N}\right) \\
& \left.-\frac{1}{12} e^{\kappa \sigma} H_{M N P} H^{M N P}-G_{\alpha \beta}(\Phi) D_{M} \Phi^{\alpha} D^{M} \Phi^{\beta}-\frac{8}{\kappa^{4}} e^{-\kappa \sigma / 2} v(\Phi)\right],
\end{aligned}
$$

where $\left\{g_{M N}, H_{3}=\mathrm{d} B_{2}+A_{1} \wedge F_{2}, \sigma\right\}$ are the bosonic fields in the gravity multiplet; $F_{M N}$ are the gauge-multiplet field strengths for $\mathcal{G} \times U(1)_{R}$; and $\Phi^{\alpha}$ denote the hyper-multiplet scalars. The dependence of the scalar potential on $\Phi^{\alpha}$ is such that its minimum is at $\Phi^{\alpha}=0$ where $v(0)=g_{1}^{2}$, and so we fix henceforth $\Phi^{\alpha}=0$. Here $g_{1}$ is the $U(1)_{R}$ gauge coupling, and we use $g$ for the $\mathcal{G}$ coupling constants 5

Using $\Phi^{\alpha}=0$ the remaining equations of motion (EOMs) become

$$
\begin{aligned}
& \frac{1}{\kappa^{2}} R_{M N}=\frac{1}{4} \partial_{M} \sigma \partial_{N} \sigma+\frac{1}{2} e^{\kappa \sigma / 2} \operatorname{Tr}\left(F_{M P} F_{N}{ }^{P}\right)+\frac{1}{4} e^{\kappa \sigma} H_{M P Q} H_{N}{ }^{P Q}-\frac{1}{4 \kappa} g_{M N} \square \sigma, \\
& \frac{1}{\kappa} \square \sigma=\frac{1}{4} e^{\kappa \sigma / 2} \operatorname{Tr}\left(F_{M N} F^{M M}\right)+\frac{1}{6} e^{\kappa \sigma} H_{M N P} H^{M N P}-\frac{8 g_{1}^{2}}{\kappa^{4}} e^{-\kappa \sigma / 2}, \\
& D_{M}\left(e^{\kappa \sigma / 2} F^{M N}\right)=\frac{\kappa}{2} e^{\kappa \sigma} H^{N P Q} F_{P Q}, \\
& D_{M}\left(e^{\kappa \sigma} H^{M N P}\right)=0,
\end{aligned}
$$

where

$$
\begin{aligned}
& A_{M}=A_{M}^{I} T_{I}, \quad F_{M N}=F_{M N}^{I} T_{I} \\
& F_{M N}^{I}=\partial_{M} A_{N}^{I}-\partial_{N} A_{M}^{I}+g c^{I}{ }_{J K} A_{M}^{J} A_{N}^{K} \\
& D_{M}=\nabla_{M}-i g A_{M}^{I} T_{I}
\end{aligned}
$$

with $\nabla_{M}$ the Lorentz covariant derivative, and $T_{I}$ are the gauge group generators with structure constants $c_{J K}^{I}$.

\footnotetext{
${ }^{4}$ For fermionic terms see [13].

${ }^{5}$ In general, if $\mathcal{G}$ consists of several simple factors, $g$ represents a collection of independent gauge couplings.
} 


\subsection{Background solutions}

The solutions to these equations whose stability is of interest are monopole-supported extra dimensions, in which the extra dimensions are supported against gravitational collapse by having a gauge flux thread the extra dimensions. Our interest in particular lies in the case where this background flux lies within the non-Abelian part of the gauge group. A broad class of these have the form [15],

$$
\begin{aligned}
& \mathrm{d} s^{2}=g_{M N} \mathrm{~d} x^{M} \mathrm{~d} x^{N}=\rho \eta_{\mu \nu} \mathrm{d} x^{\mu} \mathrm{d} x^{\nu}+\frac{\mathrm{d} \rho^{2}}{h(\rho)}+h(\rho) \mathrm{d} \phi^{2} \\
& A_{ \pm}=\frac{q^{a} Q_{a}}{2}\left(\frac{1}{\rho^{2}}-\frac{1}{\rho_{ \pm}^{2}}\right) \mathrm{d} \phi \\
& \kappa \sigma=2 \ln \rho, \quad H_{M N P}=0
\end{aligned}
$$

with

$$
\begin{aligned}
h(\rho) & =\frac{2 M}{\rho}-\frac{2 g_{1}^{2} \rho}{\kappa^{2}}-\frac{\kappa^{2} \gamma_{a b} q^{a} q^{b}}{8 \rho^{3}} \\
& =-\frac{2 g_{1}^{2}}{\kappa^{2} \rho^{3}}\left(\rho^{2}-\rho_{+}^{2}\right)\left(\rho^{2}-\rho_{-}^{2}\right),
\end{aligned}
$$

where $Q_{a}$ are the generators of the Cartan subalegbra of the Lie algebra associated with the group $\mathcal{G}$, normalized so that $\operatorname{Tr}\left(Q_{a} Q_{b}\right)=\gamma_{a b}=\gamma^{2} \delta_{a b}$, for constant $\gamma$. The $q^{a}$ identify the magnitude and direction of the background flux in the Lie algebra of $\mathcal{G}$. Finally, $\rho_{-}<\rho<\rho_{+}$, where

$$
\rho_{ \pm}=\frac{\kappa^{2}}{2 g_{1}^{2}}\left[M \pm \sqrt{M^{2}-\frac{1}{4} \gamma_{a b} q^{a} q^{b}}\right],
$$

denote the two positive values where $h\left(\rho_{ \pm}\right)=0$, at which point the geometry has a conical singularity, with deficit angle

$$
\delta_{ \pm}=1-\frac{1}{2}\left|h^{\prime}\left(\rho_{ \pm}\right)\right|=1-\frac{2 g_{1}^{2}}{\kappa^{2} \rho_{ \pm}}\left(\rho_{+}^{2}-\rho_{-}^{2}\right) .
$$

As shown in ref. [16], it is the property that these singularities are conical that defines these solutions, eqs. (2.4) and (2.5), as special cases of the more general solutions of ref. [14].

We regard the conical singularities as indicating the presence of source codimension-two branes having tensions $T_{ \pm}$,

$$
S_{\text {brane }}=-T_{ \pm} \int d^{4} y \sqrt{-\gamma_{ \pm}},
$$

with $y^{i}$ being coordinates on the brane world-volume, and $\gamma_{i j}$ the induced metric there. The tension is related to the geometry's conical defect angle through $T_{ \pm}=2 \delta_{ \pm} / \kappa^{2}$, and this connection allows us to trade the integration parameters $M$ and $q^{2}=\gamma_{a b} q^{a} q^{b}$ for the two source brane tensions. It turns out that only one combination of these parameters is fixed, and that the tensions of the branes are related to each other by a constraint [15] (see later sections for a recap of some of these features). 
These solutions break supersymmetry, apart from the special rugby-ball case where the dilaton is a constant: $\partial_{m} \sigma=0$. Whether supersymmetry breaks even in this case depends on the boundary conditions at the branes [17], which is governed by more model-dependent details of the branes themselves.

The amplitude, $q^{a}$, of the gauge field is also constrained by topology to be quantized, as follows. In order for the gauge field potential to be well-defined at $\rho_{ \pm}$, we need to cover the internal manifold with two coordinate patches. Requiring that the two patches be related by a single valued gauge transformation on their overlap leads to the following Dirac quantization condition

$$
-g e_{a I} \frac{q^{a}}{2}\left(\frac{1}{\rho_{+}^{2}}-\frac{1}{\rho_{-}^{2}}\right)=N_{I},
$$

where $N_{I}$ are integer monopole numbers, one for each gauge generator $T_{I}$. The quantities $e_{a I}$ denote the $Q_{a}$ charge of generator $T_{I}$, defined in the adjoint representation by choosing a basis of generators that satisfies $\left[Q_{a}, T_{I}\right]=e_{a I} T_{I}$ (no sum). This clearly vanishes for all generators of the Cartan subalgebra, $e_{a b}=-e_{b a}=0$. For those $T_{i}$ not in the Cartan subalgebra 6 hermitian conjugation reverses the sign of this charge, so we choose notation so that $T_{i}^{\dagger}=T_{-i}$.

\section{Rugby Balls and Spheres:}

Ref. [16] shows that these solutions go over to the unwarped rugby-ball solutions [18], when $T_{-} \rightarrow T_{+}$(and to the spherical Salam-Sezgin solutions [1] when $T_{+}=T_{-}=0$ ). In these limits, a change of coordinates puts the background into the familiar form of a spherical geometry supported by a Dirac monopole:

$$
\begin{aligned}
& \mathrm{d} s^{2}=\eta_{\mu \nu} \mathrm{d} x^{\mu} \mathrm{d} x^{\nu}+a^{2}\left(\mathrm{~d} \theta^{2}+\sin ^{2} \theta \mathrm{d} \phi^{2}\right) \\
& A_{ \pm}=-\frac{q^{a} Q_{a}}{2}(\cos \theta \mp 1) \mathrm{d} \phi \\
& \kappa \sigma=H_{M N P}=0
\end{aligned}
$$

In this case the equations of motion fix the radius of the sphere. 7$] a=\kappa /\left(2 \sqrt{2} g_{1}\right)$, and fix the monopole strength

$$
q^{2}=\gamma^{2} \delta_{a b} q^{a} q^{b}=\frac{1}{g_{1}^{2}} .
$$

(Any other value for the monopole strength would induce warping in the non-compact directions, which requires $T_{+} \neq T_{-}$). On the other hand, the Dirac quantization condition in this case reduces to:

$$
-g q^{a} e_{a I}=N_{I}
$$

and so consistency between this and eq. (2.11) in general requires relations between the otherwise independent couplings $g_{1}$ and $g$. In the simplest case where the monopole is aligned in the $U(1)_{R}$ direction [1] we have $g=g_{1}$ and consistency between equations of motion and Dirac quantisation imply the monopole number must be $N= \pm 1$.

\footnotetext{
${ }^{6}$ In the Cartan-Weyl basis of generators we label the Lie algebra of $\mathcal{G}$ by: $\left\{T_{I}\right\}=\left\{Q_{a}, T_{i}, T_{-i}\right\}$.

${ }^{7}$ More generally there is a flat direction along which the values of $\sigma$ and $a$ are correlated.
} 


\section{A concrete example}

It is useful in what follows to have in mind a concrete example that is simple enough to solve explicitly yet complicated enough to display the instabilities of later interest. For this purpose we focus on the subsector of the theory for which the gauge fields lie within a subgroup $\hat{\mathcal{G}}=S U(3) \times U(1)_{R}$ of the full group, with all hyper-scalars either neutral under the non-Abelian subgroup or transforming in the adjoint 8 and all other fields required for anomaly cancellation, including the Kalb-Ramond fields $H_{M N P}$, set to zero.

The Cartan subalgebra of $S U(3)$ is two-dimensional, $Q_{a}$ with $a=1,2$, and with the normalisation condition $\gamma_{a b}=\operatorname{Tr}\left(Q_{a} Q_{b}\right)=\frac{1}{6} \delta_{a b}\left(\right.$ so $\left.\gamma^{2}=\frac{1}{6}\right)$, these may be written

$$
Q_{1}=\frac{1}{2 \sqrt{3}}\left(\begin{array}{ccc}
1 & & \\
& -1 & \\
& & 0
\end{array}\right) \quad Q_{2}=\frac{1}{6}\left(\begin{array}{ccc}
1 & & \\
& 1 & \\
& & -2
\end{array}\right) \text {. }
$$

The remaining six generators can be divided into three pairs, $T_{i}$ and $T_{-i}$ with $i=1,2,3$, having opposite charges. The independent nonzero charge eigenvalues, $e_{a i}$, then become

\begin{tabular}{c||ccc} 
& $T_{1}$ & $T_{2}$ & $T_{3}$ \\
\hline \hline$Q_{1}$ & $\frac{1}{\sqrt{3}}$ & $\frac{1}{2 \sqrt{3}}$ & $-\frac{1}{2 \sqrt{3}}$ \\
$Q_{2}$ & 0 & $\frac{1}{2}$ & $\frac{1}{2}$
\end{tabular}

Table 1: Table of charges for adjoint fields in $S U(3)$.

The monopole breaks the $S U(3)$ gauge group down to either $U(1)_{1} \times U(1)_{2}$ or $S U(2) \times$ $U(1)_{2}$, depending on whether or not all of the eigenvalues of $q^{a} Q_{a}$ are distinct or if two of them are equal.

The case $S U(3) \rightarrow S U(2) \times U(1)_{2}:$

If two eigenvalues of $q^{a} Q_{a}$ are equal then an $S U(3)$ rotation can be performed to ensure that $q^{a} Q_{a}$ points purely in the $q^{2}$ direction 9 The spectrum of $S U(3)$ gauge bosons then decomposes into the four massless gauge fields of the unbroken gauge group together with an $S U(2)$ doublet of massive charged states, having charge $\frac{1}{2}$ with respect to $U(1)_{2}$ (and their conjugates). The Dirac quantisation condition then requires that $q^{2}=2 \mathrm{~N} / \mathrm{g}$ where $N=N_{i=2}=N_{i=3}$ is an arbitrary integer, while $N_{a}=N_{i=1}=0$.

The case $S U(3) \rightarrow U(1)_{1} \times U(1)_{2}:$

Alternatively, if all eigenvalues of $q^{a} Q_{a}$ are distinct then both $q^{1}$ and $q^{2}$ are nonzero. The $S U(3)$ gauge fields then decompose into two massless gauge fields, together with three complex massive vectors with $U(1)_{1} \times U(1)_{2}$ charges as given by Table 1: $\left(\frac{1}{\sqrt{3}}, 0\right),\left(\frac{1}{2 \sqrt{3}}, \frac{1}{2}\right)$,

\footnotetext{
${ }^{8}$ Although the hyper-scalars vanish in the background, the charge of their fluctuations plays a role in the Dirac quantization conditions.

${ }^{9}$ The same can sometimes also be done if its eigenvalues all differ, but the required $S U(3)$ transformation is then singular, a distinction that turns out not to be important for identifying which monopoles are topologically stable [4].
} 
$\left(-\frac{1}{2 \sqrt{3}}, \frac{1}{2}\right)$. The Dirac quantisation condition then requires that $q^{1}=2 \sqrt{3} s^{1} / g$ and $q^{2}=$ $2 s^{2} / g$, where $s^{1}=\frac{1}{2}\left(N_{i=3}-N_{i=2}\right)=\frac{1}{2} N_{i=1}$ and $s^{2}=\frac{1}{2}\left(N_{i=3}+N_{i=2}\right)$ are half-integer valued.

Different quantum numbers $\left(s^{1}, s^{2}\right)$ do not always label distinct monopoles. For instance if $\left(s^{1}, s^{2}\right)=\left(\frac{1}{2}, \frac{1}{2}\right)$, then

$$
g q^{a} Q_{a}=\sqrt{3} Q_{1}+Q_{2}=\frac{1}{3}\left(\begin{array}{ccc}
2 & & \\
& -1 & \\
& & -1
\end{array}\right)
$$

and so equals $-2 Q_{2}$ up to a permutation of the axes. This shows that the $\left(s^{1}, s^{2}\right)=\left(\frac{1}{2}, \frac{1}{2}\right)$ monopole is physically equivalent to the $\left(s^{1}, s^{2}\right)=(0,-1)($ or $N=-1) S U(2) \times U(1)_{2^{-}}$ preserving monopole.

\subsection{Linearised instability}

Linearised stability analysis shows that spacetimes stabilised by monopoles embedded into non-abelian groups (as above) are unstable, as we now summarise following ref. [2]. Consider therefore linearising about the background geometry

$$
\bar{g}_{M N} \mathrm{~d} x^{M} \mathrm{~d} x^{N}:=e^{\bar{A}} \eta_{\mu \nu} \mathrm{d} x^{\mu} \mathrm{d} x^{\nu}+e^{\bar{A}} \mathrm{~d} u^{2}+e^{\bar{B}} \mathrm{~d} \phi^{2}
$$

where the extra-dimensional coordinates are $\left\{x^{m}\right\}=\{u(\rho), \phi\}$. Denote the Ricci tensor for this geometry by $\bar{R}_{M N}$, and the background gauge field by $\mathcal{A}_{M}$, with field strength $\mathcal{F}_{M N}$.

The unstable tachyonic directions turn out to be among the Kaluza-Klein (KK) modes of the non-abelian gauge field that live in the extra dimensions and lie along directions of the gauge algebra that are charged under the generator along which the background monopole points:

$$
\begin{aligned}
\delta A_{u}^{I} T_{I} & :=V_{u}^{I} T_{I}=V_{u} \\
\delta A_{\phi}^{I} T_{I} & :=V_{\phi}^{I} T_{I}=V_{\phi} .
\end{aligned}
$$

Raising and lowering all indices with the rescaled background metric, $\hat{g}_{M N}=e^{\kappa \sigma / 2} \bar{g}_{M N}$, ref. [2] shows that the part of the action that is bilinear in these unstable gauge-field fluctuations is (in light-cone gauge):

$$
\begin{aligned}
S_{2}(V, V)=-\frac{1}{2} \int d^{6} X & \sqrt{-\hat{g}} \operatorname{Tr}\left[\partial_{\mu} V_{m} \partial^{\mu} V^{m}+D_{m} V_{n} D^{m} V^{n}-2\left(\partial_{u} \hat{A}\right)^{2} V_{u}^{2}\right. \\
& \left.-2\left(\partial_{u} \hat{A}\right) V_{u} D_{m} V^{m}+\hat{R}_{m n} V^{m} V^{n}+2 g \mathcal{F}_{m n} V^{m} \times V^{n}\right],
\end{aligned}
$$

where $\hat{A}=\bar{A}+\kappa \sigma / 2$, and the covariant derivative of $V_{M}$ is defined by

$$
D_{M} V_{N}=\nabla_{M} V_{N}-i g\left[\mathcal{A}_{M}, V_{N}\right]
$$

and $\nabla_{M}$ is the Lorentz covariant derivative.

Solving the linearised equations of motion and boundary conditions obtained from this action, and requiring the resulting modes to have finite kinetic energy, leads to a discrete 
spectrum of fluctuations. Taking advantage of the axial-symmetry, make the Fourier decomposition:

$$
V_{n}(X)=\sum_{\mathbf{m}} V_{n \mathbf{m}}(x, u) e^{i \mathbf{m} \phi}
$$

with $\mathbf{m}$ an arbitrary integer, $-\infty<\mathbf{m}<\infty$. To diagonalise the mode functions make the field redefinitions

$$
V_{ \pm \mathbf{m}}(x, u)=\frac{1}{\sqrt{2}}\left(e^{(\hat{A}+\hat{B}) / 4} V_{u \mathbf{m}}(x, u) \pm i e^{(3 \hat{A}-\hat{B}) / 4} V_{\phi \mathbf{m}}(x, u)\right)
$$

and perform a Kaluza-Klein decomposition

$$
V_{ \pm}(x, u)=V_{ \pm}(x) \psi_{ \pm}(u)
$$

The solutions for $\psi_{ \pm}(\rho)$ can then be found explicitly in terms of hypergeometric functions. For $\mathbf{n}=0,1,2, \ldots$ the corresponding $\mathrm{KK}$ mass spectrum for $V_{+}^{I}$ is

- For $\mathbf{m} \leq-\frac{1}{\eta_{+}}$and $\mathbf{m} \leq N_{I}+\frac{1}{\eta_{-}}$

$$
M^{2}=\frac{1}{a^{2}}\left\{\mathbf{n}(\mathbf{n}+1)-\left(\mathbf{n}+\frac{1}{2}\right)\left[\mathbf{m} \eta_{+}+\left(\mathbf{m}-N_{I}\right) \eta_{-}\right]+\mathbf{m}\left(\mathbf{m}-N_{I}\right) \eta_{+} \eta_{-}\right\} .
$$

- For $-\frac{1}{\eta_{+}}<\mathbf{m} \leq N_{I}+\frac{1}{\eta_{-}}$

$$
M^{2}=\frac{1}{a^{2}}\left\{\left(\mathbf{n}+\frac{3}{2}\right)^{2}-\frac{1}{4}+\left(\mathbf{n}+\frac{3}{2}\right)\left[\mathbf{m} \eta_{+}-\left(\mathbf{m}-N_{I}\right) \eta_{-}\right]\right\} .
$$

- For $N_{I}+\frac{1}{\eta_{-}}<\mathbf{m} \leq-\frac{1}{\eta_{+}}$

$$
M^{2}=\frac{1}{a^{2}}\left\{\mathbf{n}(\mathbf{n}-1)-\left(\mathbf{n}-\frac{1}{2}\right)\left[\mathbf{m} \eta_{+}-\left(\mathbf{m}-N_{I}\right) \eta_{-}\right]\right\} .
$$

- For $\mathbf{m}>-\frac{1}{\eta_{+}}$and $\mathbf{m}>N_{I}+\frac{1}{\eta_{-}}$

$$
M^{2}=\frac{1}{a^{2}}\left\{\mathbf{n}(\mathbf{n}+1)+\left(\mathbf{n}+\frac{1}{2}\right)\left[\mathbf{m} \eta_{+}+\left(\mathbf{m}-N_{I}\right) \eta_{-}\right]+\mathbf{m}\left(\mathbf{m}-N_{I}\right) \eta_{+} \eta_{-}\right\} .
$$

In these expressions the parameters, $\eta_{ \pm}$, are related to the two conical defect angles, $\delta_{ \pm}$, by $\eta_{ \pm}=\left(1-\delta_{ \pm} / 2 \pi\right)^{-1}$. The spectrum for $V_{-}^{I}$ is obtained from the above by transforming $\mathbf{m} \rightarrow-\mathbf{m}$ and $N_{I} \rightarrow-N_{I}$. The integer $N_{I}$ is the quantity appearing in the Dirac quantisation condition, eq. (2.9) 10

\footnotetext{
${ }^{10}$ In the sphere limit, the spectrum can be put into the familiar form $M^{2}=\frac{1}{a^{2}}\left[l(l+1)-\left(\frac{N_{I}}{2}\right)^{2}\right]$ with multiplicity $2 l+1$, where, for $V_{ \pm}^{I}, l=k+\left|1 \pm N_{I} / 2\right|$, and $k=0,1,2, \ldots$
} 
Using these expressions it is possible to show that the necessary and sufficient condition for the absence of tachyonic modes, assuming brane tensions not less than zero, is

$$
\left|N_{I}\right| \leq 1 \quad \text { for every } I
$$

For fields with $N_{I} \geq 2$ the tachyonic modes are those with $\mathbf{m}=1,2, \ldots, N_{I}-1$, while for fields $N_{I} \leq-2$ the tachyonic modes are those with $\mathbf{m}=-1,-2, \ldots, N_{I}+1$. Notice that because $\mathbf{m} \neq 0$ the instability towards growth of these modes spontaneously breaks the axially symmetry of the background.

In fact, it has long been known that non-abelian monopoles in pure gauge theory in 4 dimensions are also generically unstable, with only one dynamically stable monopole existing within each topological class [3, 5]. Similar instabilities were also found soon after in higher dimensions, compactified on spheres, both for Yang-Mills and for Einstein-Yang-Mills theories [6, 7]. We use these related instabilities in subsequent sections to try to identify the new state towards which the system evolves once the instability develops.

\section{The $S U(3) \times U(1)_{R}$ example}

It is instructive to apply this to the specific example considered above, where the monopole is embedded into $S U(3) \times U(1)_{R}$.

The case $S U(3) \rightarrow S U(2) \times U(1)_{1}:$

In this case we had $\left(q^{1}, q^{2}\right)=(2 / g)(0, N)$, with $N_{a}=N_{i=1}=0$ and $N_{i=2}=N_{i=3}=N$. In this case we find no tachyonic modes when $N=0, \pm 1$, but instability when $|N| \geq 2$ (for which there are two complex tachyonic modes, $V^{i=2}$ and $\left.V^{i=3}\right)$.

The case $S U(3) \rightarrow U(1)_{1} \times U(1)_{2}:$

Consider the monopole with $\left(s^{1}, s^{2}\right)=(1,0)$, for example, where solving for the $N_{I}$ 's leads to the nonzero values $N_{i}=(2,1,-1)$. Since $N_{i=1}=2$ this monopole has one complex unstable tacyonic direction. Similarly, the monopole with $\left(s^{1}, s^{2}\right)=(2,0)$ has tachyons amongst all three of its charged fluctuations, since $N_{i}=(4,2,-2)$. However, the embedding $\left(s^{1}, s^{2}\right)=\left(\frac{1}{2}, \frac{1}{2}\right)$ turns out to give monopole numbers $N_{i}=(1,1,0)$, and so is stable, as it must be given that it is equivalent to the $N=-1$ monopole of the $S U(2) \times U(1)_{2}$-preserving category. Since this (and its conjugate) is the only stable monopole of this category, we see that the only three stable cases preserve $S U(2) \times U(1)_{2}$, with $N=0, \pm 1$.

\subsection{Topology}

Since topological charge can cause stability for some configurations, it is worth identifying how these charges are classified for non-abelian monopoles. In the present instance the topology of the internal manifold is that of a sphere, with Euler number $\chi=2$. This is not changed by the presence of the conical defects, since the contribution to $\chi$ from the singularities compensates for the reduction that the angular defect causes in the contribution to $\chi$ from the integral of $R$ over the internal space.

The non-trivial topology associated with the Dirac monopole embedded into the gauge group $\mathcal{G}$ is similarly classified by $\pi_{1}(\mathcal{G})$. This can be seen explicitly in the so called Wu-Yang 


\begin{tabular}{c|c|c} 
Gauge group $\mathcal{G}$ & $\pi_{1}(\mathcal{G})$ & Center \\
\hline$S U(N)$ & 1 & $Z_{N}$ \\
$S O(2 k+1)$ & $Z_{2}$ & $Z_{2}$ \\
$S O(4 k)$ & $Z_{2}$ & $Z_{2} \times Z_{2}$ \\
$S O(4 k+2)$ & $Z(k=0), Z_{2}(k \geq 1)$ & $Z_{4}$ \\
$S p(N)$ & 1 & $Z_{2}$ \\
$E_{8}$ & 1 & 1 \\
$E_{7}$ & 1 & $Z_{2}$ \\
$E_{6}$ & 1 & $Z_{3}$ \\
$F_{4}$ & 1 & 1 \\
$G_{2}$ & 1 & 1
\end{tabular}

Table 1: $\pi_{1}$ and centres for the simple Lie groups.

construction [19], used above, wherein the extra dimensions are covered with two patches, each with a non-singular gauge potential $A_{ \pm}$. In this case the $A_{ \pm}$must differ on the overlap of the two patches at the equator by a single-valued gauge transformation, and so defines a map from $S^{1}$ to $\mathcal{G}$ that is classified by $\pi_{1}(\mathcal{G}) .11$

For non-abelian groups the integer corresponding to this topological classification can be written explicitly in terms of the representative gauge fields. Suppose for example, the gauge algebra is $S U(N)$, and all charged fields transform in the adjoint representation. Then the global group is actually $\mathcal{G}=S U(N) / \mathbb{Z}_{N}$ because the adjoint representation uses the same matrix to represent two group elements that differ only by an element of the group's center. Define the magnetic flux, $\Phi$, using the following integral,

$$
\Phi=\frac{1}{N} \operatorname{Tr} \exp \left[i g \oint \mathrm{d} s\left(A_{+M}-A_{-M}\right) \frac{\mathrm{d} x^{M}}{\mathrm{~d} s}\right],
$$

where the path is taken as the closed loop around the equator in the overlap of the two patches on which the two gauge configurations, $A_{+}$and $A_{-}$, are respectively defined. This expression, when evaluated using explicit gauge configurations, produces a phase

$$
\Phi=\exp \left(\frac{2 \pi i L}{N}\right)
$$

where $0 \leq L<N$ is the integer that labels the corresponding element of $\pi_{1}\left(S U(N) / Z_{N}\right)=$ $Z_{N}$.

Table 1 gives $\pi_{1}$ and the centers of all the simple Lie algebras. Amongst the known anomaly-free non-abelian gauge groups in 6D chiral supergravity, those involving non-Abelian

\footnotetext{
${ }^{11}$ If Higgs fields spontaneously break $\mathcal{G} \rightarrow \mathcal{H}$, then magnetic charge would instead be classified by $\pi_{1}(\mathcal{H})$. For smooth configurations without Dirac strings this reduces to the subgroup of $\pi_{1}(\mathcal{H})$ consisting of those elements which are contractible in $\mathcal{G}$, denoted $\pi_{1}(\mathcal{H})_{\mathcal{G}}$. This is equivalent to the familiar classification of non-singular monopoles by $\pi_{2}(\mathcal{G} / \mathcal{H})$ (interpreted as classifying the map from the $S_{2}$ at spatial infinity to the vacuum manifold $\mathcal{G} / \mathcal{H}$ ) due to the isomorphism $\pi_{1}(\mathcal{H})_{\mathcal{G}} \sim \pi_{2}(\mathcal{G} / \mathcal{H})$. Similarly, in the absence of a Higgs contribution to topological charge the classification $\pi_{1}(\mathcal{H})$ reduces to the Wu-Yang classification $\pi_{1}(\mathcal{G})$, since $\pi_{1}(\mathcal{H}) / \pi_{1}(\mathcal{H})_{\mathcal{G}} \sim \pi_{1}(\mathcal{G})$.
} 
gauge groups with non-trivial topology are the classic $E_{7} \times E_{6} \times U(1)_{R}$ model [20], for which all hyper-multiplets are singlets under $E_{6}$; as well as two models by Avramis and Kehaghias [21], respectively involving $E_{6}$ (with hyper-multiplets only in the adjoint representation) or $S O(N)$ 's.

The example $\mathcal{G}=S U(3) \times U(1)_{R}$

For the Dirac monopole embedded in $S U(3)$, with all fields charged under the $S U(3)$ subgroup transforming in the adjoint representation, as described above the global group is actually $\hat{\mathcal{G}}=\left[S U(3) / \mathbb{Z}_{3}\right] \times U(1)_{R}$, and the topological classification is given by:

$$
\begin{aligned}
\pi_{1}(\hat{\mathcal{G}}) & =\pi_{1}(\mathcal{G})+\pi_{1}\left(U(1)_{R}\right) \\
& =\pi_{1}\left(S U(3) / \mathbb{Z}_{3}\right)+\pi_{1}\left(U(1)_{R}\right) \\
& =\mathbb{Z}_{3}+\mathbb{Z}
\end{aligned}
$$

Notice that each simple factor of $\hat{\mathcal{G}}$ gives rise to its own topological classification, provided that the monopole lies at least partially in the factor of interest.

If the monopole is embedded purely within the $S U(3)$ then the topology is simply classified by $\pi_{1}\left(S U(3) / \mathbb{Z}_{3}\right)=\mathbb{Z}_{3}$. In the case where the monopole preserves an unbroken $S U(2) \times U(1)_{2}$ we have seen that it can always be written as 12

$$
\begin{gathered}
\qquad A_{ \pm}=-\frac{1}{2} \mathbb{M}(\cos \theta \mp 1) \mathrm{d} \phi \\
\text { where } \quad \mathbb{M}=2 N Q_{2}=\frac{N}{3}\left(\begin{array}{ccc}
1 & & \\
& 1 & \\
& & -2
\end{array}\right)
\end{gathered}
$$

and, as before, $Q_{2}$ is the second Cartan generator of $S U(3)$ while $N$ is the integer of the Dirac quantisation condition. In this case the associated flux evaluates to $\Phi=e^{i 2 \pi N / 3} \in \mathbb{Z}_{3}$, showing that it is $L=N(\bmod 3)$ that labels the distinct topological class [4]. The finding that linearised stability requires $N=0, \pm 1$ is therefore consistent with the expectation that there is only one stable monopole in each of the three independent topological sectors.

\section{The Instability's Endpoint}

The previous sections argue that monopole-supported flux compactifications in 6D supergravity are generically unstable, provided the monopole is embedded within a non-Abelian factor of the gauge group. We now ask what the new configuration is towards which such an unstable non-Abelian monopole evolves.

As mentioned above, this problem is well understood in the case of pure Yang-Mills (YM) theory, where the instability describes the decay into the lightest monopole within the given topological class [5]. Our goal is to address what such a decay implies when the monopole in question supports an extra-dimensional compactification. We do so in this section starting

\footnotetext{
${ }^{12}$ More generally, a monopole in $S U(3)$ can always be written as $g A_{ \pm}=-\frac{1}{2} \mathbb{M}(\cos \theta \mp 1) \mathrm{d} \phi$ where $\mathbb{M}=2 L Q_{2}+\operatorname{diag}\left(r_{1}, r_{2}, r_{3}\right), L$ is one of $0,1,2$ and $r_{1}, r_{2}, r_{3}$ are integers that sum to zero [4].
} 
with simple spherical compactifications of extra-dimensional Yang Mills and Einstein-YangMills (EYM) systems (including a cosmological constant, $\Lambda$ ). We defer the qualitatively different case of EYM-Dilaton theories relevant to higher-dimensional supergravity to the next section.

Concretely, consider the unstable $N=2 S U(3)$ monopole described in previous sections, for which $g\left(q^{1}, q^{2}\right)=(0,4)$. Because $N \neq 0(\bmod 3)$, this state has a non-trivial topology that prevents it from decaying into a topologically trivial configuration. It must instead decay into the stable monopole with $N=-1$, doing so by emitting magnetic radiation (see e.g. [3, 5]).

\subsection{Einstein Yang-Mills Theory}

When the decaying monopole supports a compactified extra dimension, its decay should also cause the extra-dimensional geometry to change. But since the decay also reduces the 4D monopole energy density, its decay should also change the curvature of the large dimensions we observe. We first show how this takes place in detail, working within the Einstein-YangMills system. In this case, we must solve both the Einstein and Maxwell equations of motion to check that the expected stable monopole is a possible endpoint solution.

Consider then six-dimensional gravity coupled to a Yang-Mills field and a positive 6D cosmological constant, $\Lambda$. We start with a solution, $\operatorname{Mink}_{4} \times S_{2}$, for this system comprising an unstable $S U(3)$ monopole supporting two spherical extra dimensions, with $\Lambda$ adjusted to allow the observable four dimensions to be flat. This initial monopole then decays into the topologically connected stable monopole as above, whilst the background geometry appropriately adjusts itself. We do not try to follow the time-dependence of this process in its full transient glory. Instead we directly seek the endpoint solution to which it ultimately evolves, under the assumption that this endpoint also remains maximally symmetric in the 4 visible and 2 internal dimensions.

It turns out that we are led in this way to two possible endpoint solutions. Either the internal sphere shrinks whilst the non-compact directions curve into anti-de Sitter space (AdS), or the sphere grows whilst the 4D spacetime curves into a de Sitter (dS) universe. Based on the principle that the evolution lowers the effective 4D scalar potential energy, we expect it is the AdS solution towards which the system evolves.

\section{Endpoint Solutions}

We start with the 6D EYM action,

$$
S_{E Y M}=\frac{1}{\kappa^{2}} \int d^{6} x \sqrt{-g}\left[R-\frac{\kappa^{2}}{4} \operatorname{Tr}\left(F_{M N} F^{M N}\right)-\Lambda\right],
$$

containing only gravity, $g_{M N}$, the Yang-Mills field, $A_{M}$, and the $6 \mathrm{D}$ positive cosmological constant, $\Lambda$. The equations of motion for this system become

$$
\begin{aligned}
& R_{M N}=\frac{\kappa^{2}}{2} \operatorname{Tr}\left(F_{M P} F_{N}{ }^{P}\right)+g_{M N}\left(\frac{\Lambda}{4}-\frac{\kappa^{2}}{16} \operatorname{Tr} F^{2}\right) \\
& \nabla_{M} F^{M N}-i g A_{M} F^{M N}=0,
\end{aligned}
$$


for which we seek solutions having maximal symmetry in both 4 large dimensions and 2 small ones

$$
\mathrm{d} s^{2}=g_{\mu \nu} \mathrm{d} x^{\mu} \mathrm{d} x^{\nu}+a^{2}\left(\mathrm{~d} \theta^{2}+\sin ^{2} \theta \mathrm{d} \phi^{2}\right)
$$

with the maximally symmetric metric, $g_{\mu \nu}$, satisfying $R_{\mu \nu}=3 \lambda g_{\mu \nu}$, with $4 \mathrm{D}$ curvature constant $\lambda$. In our conventions the cases $\lambda>0, \lambda=0$ or $\lambda<0$ respectively correspond to $\mathrm{dS}$, flat and AdS geometries.

Consider now solutions for which the Maxwell field strength only has nonzero internal components, $F_{m n}$, and depends only on the coordinate $\theta$ (as in the monopole solution). The equation of motion for the gauge field then implies

$$
F_{\theta \phi}=\frac{q^{a} Q_{a}}{2} \sin \theta
$$

where the constants $q^{a}$ again parametrise the monopole strength. Recalling our convention $\operatorname{Tr}\left(Q_{a} Q_{b}\right)=\gamma_{a b}=\gamma^{2} \delta_{a b}$, we find after use of the 4D components of Einstein's equations,

$$
R_{\mu \nu}=3 \lambda g_{\mu \nu}=g_{\mu \nu}\left(\frac{\Lambda}{4}-\frac{\kappa^{2} q^{2}}{32 a^{4}}\right)
$$

where $q^{2}=\gamma_{a b} q^{a} q^{b}$. From the 2D Einstein equations we instead find

$$
R_{m n}=\frac{g_{m n}}{a^{2}}=g_{m n}\left[\frac{3 \kappa^{2} q^{2}}{32 a^{4}}+\frac{\Lambda}{4}\right],
$$

leading to the following conditions for $\lambda$ and $a$ :

$$
\begin{aligned}
3 \lambda & =\left(\frac{\Lambda}{4}-\frac{\kappa^{2} q^{2}}{32 a^{4}}\right) \\
\frac{1}{a^{2}} & =\frac{3 \kappa^{2} q^{2}}{32 a^{4}}+\frac{\Lambda}{4}=\frac{\kappa^{2} q^{2}}{8 a^{4}}+3 \lambda .
\end{aligned}
$$

If we choose the initial monopole to be the unstable configuration having $q^{a}=q_{i}^{a}=$ $(0,4 / g)$, then $q_{i}^{2}=16 \gamma^{2} / g^{2}$. It is for this configuration that we tune the $6 \mathrm{D}$ cosmological constant to obtain a flat $4 \mathrm{D}$ spacetime, $\lambda=0$. This fixes the initial radius of the internal sphere and the $6 \mathrm{D}$ cosmological constant to be

$$
a_{i}^{2}=\frac{\kappa^{2} q_{i}^{2}}{8}=\frac{2 \kappa^{2} \gamma^{2}}{g^{2}} \quad \text { and } \quad \Lambda=\frac{\kappa^{2} q_{i}^{2}}{8 a_{i}^{4}}=\frac{1}{a_{i}^{2}}=\frac{g^{2}}{2 \kappa^{2} \gamma^{2}} .
$$

To find the endpoint, we now take the monopole charge to be the topologically stable one having $N=-1$ and so $q_{f}^{a}=(0,-2 / g)$, and so $q_{f}^{2}=4 \gamma^{2} / g^{2}=\frac{1}{4} q_{i}^{2}$. Since $\Lambda$ is no longer free to be adjusted, we now solve eqs. (3.38) and (3.39) for the final radius, $a_{f}$, of the 2D sphere, and the curvature, $\lambda_{f}$, of the final $4 \mathrm{D}$ spacetime.

We find in this way that the $4 \mathrm{D}$ curvature becomes

$$
3 \lambda_{f}=\frac{1}{4 a_{i}^{2}}-\frac{\kappa^{2} q_{f}^{2}}{32 a_{f}^{4}}=\frac{1}{4 a_{i}^{2}}-\frac{a_{i}^{2}}{16 a_{f}^{4}}
$$


while the new radius of the 2 -sphere is given by

$$
\frac{1}{a_{f}^{2}}=\frac{1}{4 a_{i}^{2}}+\frac{3 \kappa^{2} q_{f}^{2}}{32 a_{f}^{4}}=\frac{1}{4 a_{i}^{2}}+\frac{3 a_{i}^{2}}{16 a_{f}^{4}} .
$$

This has two roots, given by

$$
a_{f \pm}^{2}=2 a_{i}^{2}\left[1 \pm \sqrt{1-\frac{3}{4} \frac{q_{f}^{2}}{q_{i}^{2}}}\right]=2 a_{i}^{2}\left[1 \pm \frac{1}{4} \sqrt{13}\right]
$$

and so $a_{f+} \simeq 1.95 a_{i}$ while $a_{f-} \simeq 0.444 a_{i}$. The corresponding $4 \mathrm{D}$ curvature then becomes

$$
3 \lambda_{f \pm}=\frac{1}{a_{i}^{2}}\left(\frac{7 \pm 2 \sqrt{13}}{29 \pm 8 \sqrt{13}}\right)
$$

and so $\lambda_{f+} \simeq 0.0819 / a_{i}^{2}$ and $\lambda_{f-} \simeq-0.452 / a_{i}^{2}$. Clearly the radius of the sphere increases for the $\mathrm{dS}$ solution and decreases for the AdS one.

\section{Energetics}

Intuitively, one would expect the AdS case to be the natural endpoint, since one expects to obtain a negative potential energy after lowering it below the initially zero value needed to ensure a flat 4 dimension, as we next check explicitly. To this end define the potential energy (per unit $3 \mathrm{D}$ volume), $\mathcal{E}$, of the effective $4 \mathrm{D}$ theory as the sum of the static $6 \mathrm{D}$ energy (i.e. gradient, magnetic and potential energy), integrated over the extra dimensions, with

$$
\mathcal{E}=\frac{1}{\kappa^{2}} \int \mathrm{d}^{2} x \sqrt{g_{2}}\left[-R_{(2)}+\frac{\kappa^{2}}{4} \operatorname{Tr} F_{m n} F^{m n}+\Lambda\right] .
$$

Here $R_{(2)}=2 / a^{2}$ denotes the $2 \mathrm{D}$ curvature scalar, while the magnetic energy goes as $\operatorname{Tr} F_{m n} F^{m n}=q^{2} / 2 a^{4}$, leading to

$$
\begin{aligned}
\mathcal{E} & =\frac{4 \pi a^{2}}{\kappa^{2}}\left[-\frac{2}{a^{2}}+\Lambda+\frac{\kappa^{2} q^{2}}{8 a^{4}}\right] \\
& =\left(\frac{4 \pi a^{2}}{\kappa^{2}}\right)^{2} \frac{\kappa^{2}}{4 \pi}\left[-\frac{2}{a^{4}}+\frac{\Lambda}{a^{2}}+\frac{\kappa^{2} q^{2}}{8 a^{6}}\right] .
\end{aligned}
$$

The second equality of eq. (3.46) pulls out four powers of the $4 \mathrm{D}$ Planck mass, $M_{p}^{2}=$ $4 \pi a^{2} / \kappa^{2}$, which is useful when verifying that $\mathcal{E}$ as defined reproduces the correct equations of motion when used in the $4 \mathrm{D}$ field theory. It is useful to display the factors of $M_{p}$ explicitly in this way because transforming to the 4D Einstein frame ensures that these are held fixed when the $4 \mathrm{D}$ field $a$ is varied to minimize the potential energy, leading to the condition

$$
\frac{\partial\left(\mathcal{E} / M_{p}^{4}\right)}{\partial a}=\frac{2 \kappa^{2}}{\pi a^{3}}\left[\frac{1}{a^{2}}-\frac{\Lambda}{4}-\frac{3 \kappa^{2} q^{2}}{32 a^{4}}\right]=0,
$$


in agreement with eq. (3.39) determining $a$. The 4D Einstein equations similarly equate $R_{\mu \nu}=3 \lambda g_{\mu \nu}$ to $\left(\mathcal{E} / M_{p}^{2}\right) g_{\mu \nu}$, leading to the condition

$$
\begin{aligned}
6 \lambda & =-\frac{2}{a^{2}}+\Lambda+\frac{\kappa^{2} q^{2}}{8 a^{4}} \\
& =\frac{\Lambda}{2}-\frac{\kappa^{2} q^{2}}{16 a^{4}}
\end{aligned}
$$

where the last equality — which agrees with eq. (3.38) — uses the field equation, eq. (3.47), for $a$.

We may now compare the value of $\mathcal{E}$ when evaluated at the initial and final configurations considered above. Evaluating using our previous results for $a_{i}, q_{i}^{2}$ and $\Lambda$ leads to $\mathcal{E}_{i}=0$ for the initial unstable solution, consistent with having tuned $\Lambda$ to ensure the flatness of the initial 4D geometry. For the two candidate endpoint solutions, the potential energy is instead

$$
\begin{aligned}
\mathcal{E}_{f \pm} & =\frac{4 \pi}{\kappa^{2}}\left[-2+\Lambda a_{f \pm}^{2}+\frac{\kappa^{2} q_{f}^{2}}{8 a_{f \pm}^{2}}\right] \\
& =\frac{4 \pi}{\kappa^{2}}\left[-2+\left(\frac{a_{f \pm}}{a_{i}}\right)^{2}+\frac{q_{f}^{2}}{q_{i}^{2}}\left(\frac{a_{i}}{a_{f \pm}}\right)^{2}\right] \\
& =\frac{2 \pi}{\kappa^{2}}\left(\frac{14 \pm 4 \sqrt{13}}{4 \pm \sqrt{13}}\right),
\end{aligned}
$$

and so $\mathcal{E}_{f+} \simeq 3.74\left(2 \pi / \kappa^{2}\right)>0$ and $\mathcal{E}_{f-} \simeq-1.07\left(2 \pi / \kappa^{2}\right)<0$. Clearly we have $a_{f+}>a_{i}>$ $a_{f-}$, and $\mathcal{E}_{f+}>\mathcal{E}_{i}>\mathcal{E}_{f-}$, indicating that the endpoint solution reached after the instability indeed corresponds to a shrunken extra-dimensional sphere together with 4D AdS space.

\section{Endpoint Revisited: Including the Dilaton}

We next reconsider the problem of direct interest for higher dimensional supergravity, by supplementing the Einstein-YM theory with an appropriate scalar dilaton field. In this case we find the endpoint configurations do not preserve the maximal symmetry of the underlying 4D and/or 2D geometries of the original unstable monopole-supported system. As emphasised in [22], the presence of the dilaton crucially changes the dynamics of the system, and this considerably complicates the search for the new endpoint solutions.

To see why the dilaton is so different we again start with a monopole-supported solution to $6 \mathrm{D}$ chiral gauged supergravity with couplings chosen to allow a Mink $4 \times S_{2}$ solution with 4 flat large dimensions, see Eq. (2.10-2.11). Now, however, if the monopole decays to its stable topological cousin, a new maximally symmetric solution supported by the stable monopole no longer satisfies the field equations (2.2), which require $\square \sigma=0$ together with the Einstein and Yang-Mills equations. In detail, the equations of motion under the maximally symmetric ansatz $d s^{2}=\mathrm{d} s_{4}^{2}+a^{2}\left(\mathrm{~d} \theta^{2}+\sin ^{2} \theta \mathrm{d} \phi^{2}\right), F_{\theta \phi}=\frac{q^{a} Q_{a}}{2} \sin \theta$ and $\sigma=\sigma_{0}=$ const, with $\mathrm{d} s_{4}^{2}$ the metric on $4 \mathrm{D}(\mathrm{A}) \mathrm{dS}$ or Minkowski spacetime, together imply the $4 \mathrm{D}$ curvature $\lambda=0$, $a e^{-\kappa \sigma_{0} / 4}=\kappa / 2 \sqrt{2} g_{1}$ and $q^{2}=1 / g_{1}^{2}$. This is to be compared with the initial configuration Eq. 
(2.10 2.11). So, although one combination of the parameters, say $a e^{\kappa \sigma_{0} / 4}$, is left free thanks to the classical scaling symmetry, the magnetic flux in particular is fixed to its original unstable - magnitude if we insist on keeping the maximal symmetries. This shows that once the monopole flux decays the dilaton gradient, $\partial_{M} \sigma$, is necessarily nonzero, thereby picking out preferred directions in the underlying spacetime.

A key question asks whether this gradient points in the compact two directions, $\partial_{m} \sigma \neq 0$, or in the large spacetime directions, $\partial_{\mu} \sigma$. In this section we first argue that the system is likely to prefer growing nonzero gradients in the large 4 dimensions, and then describe the relative merits of two classes of candidate endpoint solutions that break the 4D spacetime symmetries: a one-parameter family of supersymmetric solutions [9]; and a class of new solutions to which one is led by adapting the arguments of the previous section to include the dilaton.

\subsection{D or 2D: Which symmetries break?}

We now argue that for 6D supergravity it is the 4D spacetime symmetries that generically prefer to break. If true this is somewhat surprising, since the instability revealed by the linearised analysis is in modes that vary in the internal 2 dimensions and not the macroscopic 4 dimensions. However, it is known [10] that all of the axially symmetric bulk solutions having AdS 4D geometry necessarily have a curvature singularity in the 2D geometry at the position of one of the two source branes 13 Any decay to a solution of the form $\mathrm{AdS}_{4} \times M_{2}$ therefore necessarily requires the development of a curvature singularity in the $2 \mathrm{D}$ geometry at the position of one of the source branes, even if the initial unstable solution has no such a singularity. But the divergence of bulk fields at a singularity is related to the physical properties of the brane which is situated there [11, 12], with a curvature singularity in particular implying a brane coupling to the bulk dilaton. Since it is not clear how such a change to intrinsic brane properties can be triggered by the decay of a monopole in the bulk, we instead explore the possibility that it is the 4D spacetime symmetries that break.

The simplest way to see the necessity for a curvature singularity is to recognize that the effective 4D potential energy turns out to depend only on the near-brane limit of the $\sigma$ field when its derivatives, $\partial_{m} \sigma$, point purely along the 2D directions [15]. That is, we evaluate

$$
\mathcal{E}=\int \mathrm{d}^{2} x \sqrt{g_{2}}\left[-\frac{1}{\kappa^{2}} R_{(2)}+\frac{1}{4} \partial_{m} \sigma \partial^{m} \sigma+\frac{1}{4} e^{\kappa \sigma / 2} \operatorname{Tr} F_{m n} F^{m n}+\frac{8 g_{1}^{2}}{\kappa^{4}} e^{-\kappa \sigma / 2}\right]
$$

at an arbitrary solution to the field equations, eqs. (2.2), assuming only that all tensor components point purely along the compact 2 dimensions. Use of the Einstein and dilaton equations in particular then show [15] that

$$
\mathcal{E}=-\frac{1}{2 \kappa} \int \mathrm{d}^{2} x \sqrt{g_{2}} \square \sigma
$$

which vanishes on a smooth manifold. For example, when evaluated for the particular

\footnotetext{
${ }^{13}$ This is also a corollary of the fact [10] that all of the solutions having only conical singularities at the branes have $4 \mathrm{D}$ geometries that are flat.
} 
solutions of eqs. (2.4) and (2.5), we find (keeping in mind the conical singularities at $\rho=\rho_{ \pm}$)

$$
\mathcal{E}=-\frac{\pi}{\kappa} \int_{\rho_{-}}^{\rho_{+}} \mathrm{d} \rho \partial_{\rho}\left[\sqrt{g_{2}} \partial^{\rho} \sigma\right]=\frac{2 \pi}{\kappa^{2}}\left[\frac{h\left(\rho_{-}\right)}{\rho_{-}}-\frac{h\left(\rho_{+}\right)}{\rho_{+}}\right]=0 .
$$

In the presence of singularities localized at source branes, each brane can be isolated within a small circle that acts as the boundary of the bulk geometry, leading the right-handside of eq. (4.52) to evaluate to a sum of terms involving the radial dilaton derivative, $n \cdot \partial \sigma$, evaluated at the brane positions. But the presence of such a nonzero scalar gradient near the codimension-2 brane requires $\phi$ to diverge logarithmically there, and the stress energy of this configuration makes the curvature also diverge. This argument is in agreement with the explicitly known solutions of ref. [10].

But the near-brane dilaton derivative is related [11, 12] by the bulk-brane matching conditions to the effective codimension- 2 brane tension, $T_{2}(\phi)$, with $n \cdot \phi$ being proportional to its derivative $T_{2}^{\prime}$. As such, the near-brane dilaton derivative cannot change without there also being a physical change to the source branes, making such a configuration an unlikely endpoint for an unstable monopole. This being said, we shall also find the necessity of new types of singularities in some solutions breaking the $4 \mathrm{D}$ symmetries, and so this argument cannot be regarded as decisive until the interpretation of those singularities is better understood.

With this motivation we next examine two categories of candidate endpoint solutions that break the $4 \mathrm{D}$ symmetries.

\subsection{Supersymmetric $\mathbf{A d S}_{3} \times \tilde{S}_{3}$}

Supersymmetric solutions are always attractive options when seeking stable endpoints from initially unstable initial configurations, and it is the remarkable scarcity of such solutions having the form $M_{4} \times M_{2}$, with $M_{4}=\mathrm{Mink}_{4}$ or $\mathrm{AdS}_{4}$, that helps make the endpoint of monopole decay in 6D supergravity such a puzzle. The only known solutions of this type have $M_{4}=$ Mink $_{4}, M_{2}=S_{2}$, and align the monopole in the $U(1)_{R}$ directions with monopole number $N= \pm 1[1]$.

Other supersymmetric solutions do exist [9], however, they just have fewer 4D spacetime symmetries. These solutions have geometries $\mathrm{AdS}_{3} \times \tilde{S}_{3}$, where $\tilde{S}_{3}$ denotes a one-parameter family of 'squashed' 3 -spheres. The field configurations have constant dilaton, $\partial_{M} \sigma=0$, and

$$
\begin{aligned}
\mathrm{d} s^{2} & =\mathrm{d} s_{\mathrm{AdS}_{3}}+a^{2}\left(\omega_{1}^{2}+\omega_{2}^{2}\right)+b^{2} \omega_{3}^{2} \\
H_{3} & =\xi\left(\omega_{1} \wedge \omega_{2} \wedge \omega_{3}+\frac{\varepsilon_{3}}{a^{2} b}\right) \\
F_{2} & =k \omega_{1} \wedge \omega_{2}
\end{aligned}
$$

where $\mathrm{d} s_{\mathrm{AdS}_{3}}^{2}$ is the line-element for $\mathrm{AdS}_{3}$ and $\varepsilon_{3}$ denotes the volume 3-form for the internal $3 \mathrm{D}$ geometry. The $\omega_{m}$ denote the left-invariant 1-forms on the 3-sphere, that satisfy $\mathrm{d} \omega_{m}=$ $-\frac{1}{2} \epsilon_{m n p} \omega_{n} \wedge \omega_{p}$, and so

$$
\omega_{1}+i \omega_{2}=e^{-i \psi}(\mathrm{d} \theta+i \sin \theta \mathrm{d} \phi), \quad \omega_{3}=\mathrm{d} \psi+\cos \theta \mathrm{d} \phi
$$

where $(\theta, \phi, \psi)$ are Euler angles on the 3-sphere. 
The equations of motion impose the following three relations among the solution's four parameters, $a, b, \xi$ and $k[9]$ :

$$
b^{2}=\kappa^{5 / 2} \xi, \quad a^{2}=\frac{\kappa^{3} k}{4 g_{1}}=\frac{1}{16 g_{1}^{2}}\left(1 \pm \sqrt{1-32 \kappa^{1 / 2} g_{1}^{2} \xi}\right)
$$

in terms of which the $\mathrm{AdS}_{3}$ Ricci tensor is $R_{\alpha \beta}=2 \lambda g_{\alpha \beta}$ with $\lambda=-b^{2} /\left(4 a^{4}\right)$.

Is this the endpoint of the evolution away from the unstable monopole? Such a scenario would be very attractive, indicating a dynamic spontaneous compactification wherein the monopole instability triggers one of the large 4 dimensions to roll up into one of the directions in $\tilde{S}_{3}$. And because $\lambda$ is negative this might be argued to be favoured energetically in terms of an appropriate 3D potential energy. Better yet, the supersymmetric $\mathrm{Mink}_{4} \times S_{2}$ solution can be obtained formally from the $\operatorname{AdS}_{3} \times \tilde{S}_{3}$ solutions by taking the limit $b \rightarrow 0$ [9], indicating there might be a plausible path through field space leading from the initial unstable configuration to the final supersymmetric one.

There are a number of possible objections to the proposal that these solutions represent to endpoint of the monopoles of present interest, however. Not least, the natural way to obtain 4 large directions from $\mathrm{AdS}_{3} \times \tilde{S}_{3}$ is by taking the lone squashed direction to become large, $b \gg a$, rather than taking $b \ll a$. However in the limit $b \gg a$ the curvature of the large 3 dimensions becomes larger and not smaller, and there is furthermore an obstruction to taking this limit within the supersymmetric solutions since it formally would require taking $\xi$ very large, but $a^{2}$ becomes complex in this limit. We therefore next seek other options for the decay endpoint.

\subsection{Deking the Dilaton}

14 As noted above, it is the dilaton that appears to prevent the system's relaxation towards a maximally symmetric solution, and so removes the attractive picture obtained in the EYM system described in $\S 3$. In the remainder of this section we use an elegant trick [23] that reformulates the EYM-dilaton system as a dilaton-free system in higher dimensions. We do so with the goal of exploring whether the analysis of $\S 3$ can lead to a better candidate endpoint, for which the preserved maximal symmetries involve the fictitious dimensions associated with the dilaton rather than being part of the physical 6 dimensions of our starting supergravity.

The idea behind the trick is that the dilaton can be regarded as a modulus obtained by compactifying a simpler system in higher dimension 15 In particular, we consider EYM theory in $(6+n)$ dimensions, chosen so that its dimensional reduction to $6 \mathrm{D}$ leads to the dilaton-EYM theory of interest. With care, solutions in the higher dimensional non-dilatonic theory can be reduced to solutions of dilatonic Einstein Yang-Mills in six dimensions, and 6D supergravity configurations can be 'oxidised' to higher dimensional solutions. Ref. [24] performs a similar analysis to study the dynamics of instabilities in warped de Sitter solutions to 6D dilatonic Einstein Maxwell theory.

\footnotetext{
${ }^{14}$ deke $v$. (in ice hockey) to draw a defending player out of position by faking a shot or movement: deked the goalie with a move from left to right.

${ }^{15} \mathrm{~A}$ similar logic underlies the discussion of $F$-theory vacua in Type IIB string compactifications having nontrivial dilaton profiles.
} 


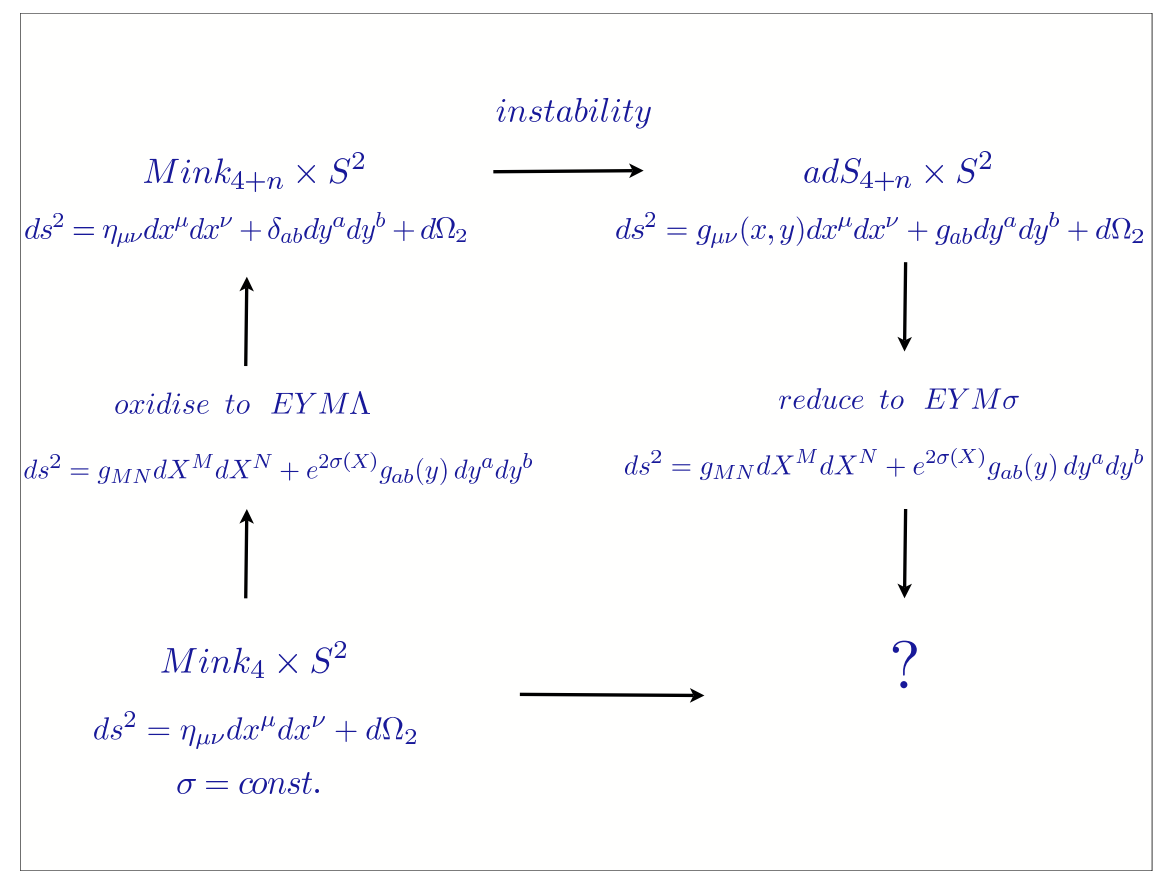

Figure 1: The oxidation-reduction cycle used to generate solutions in the 6D dilatonic theory.

This trick is useful because the stability analysis of previous sections can be translated word-for-word to the higher dimensional system, at least for unwarped backgrounds 16 In particular, an unstable monopole-supported configuration with geometry $\operatorname{Mink}_{(4+n)} \times S_{2}$, is unstable for large enough magnetic quantum numbers, and applying the arguments of $\S 3$ to the higher-dimensional system indicates a decay to $\operatorname{AdS}_{(4+n)} \times S_{2}$ supported by a stable monopole. The logic (illustrated in Fig. 1) then is to dimensionally reduce both the unstable solution and its stable endpoint down to $6 \mathrm{D}$ to find the corresponding transition to which this points in the lower-dimensional dilaton system.

\subsubsection{Oxidation/Reduction}

To proceed in detail we start with the $D=6+n$ action [23, 24]

$$
S=\frac{1}{\kappa_{D}^{2}} \int \mathrm{d}^{D} x \sqrt{-g_{D}}\left[R_{(D)}-\frac{\kappa_{D}^{2}}{4} \operatorname{Tr} \mathcal{F}^{2}-\Lambda\right]
$$

whose equations of motion are

$$
\begin{aligned}
& R_{\Lambda \Gamma}=\frac{\kappa_{D}^{2}}{2} \operatorname{Tr}\left(\mathcal{F}_{\Lambda \Omega} \mathcal{F}_{\Gamma}{ }^{\Omega}\right)+\frac{g_{\Lambda \Gamma}}{(D-2)}\left(\Lambda-\frac{\kappa_{D}^{2}}{4} \operatorname{Tr} \mathcal{F}^{2}\right) \\
& \nabla_{\Lambda} \mathcal{F}^{\Lambda \Gamma}-i g \mathcal{A}_{\Lambda} \mathcal{F}^{\Lambda \Gamma}=0 .
\end{aligned}
$$

\footnotetext{
${ }^{16}$ The bilinear action for the relevant modes is identical in this case (see equation (44) of [25]).
} 
To dimensionally reduce we seek solutions to these equations having the form 17

$$
\begin{aligned}
& \mathrm{d} s_{D}^{2}=g_{\Lambda \Gamma} \mathrm{d} x^{\Lambda} \mathrm{d} x^{\Gamma}=\hat{g}_{M N}(x) \mathrm{d} x^{M} \mathrm{~d} x^{N}+e^{2 \varphi(x)} g_{a b}(y) \mathrm{d} y^{a} \mathrm{~d} y^{b} \\
& =\quad e^{-n \varphi(x) / 2} g_{M N}(x) \mathrm{d} x^{M} \mathrm{~d} x^{N}+e^{2 \varphi(x)} g_{a b}(y) \mathrm{d} y^{a} \mathrm{~d} y^{b} \\
& \mathcal{F}_{M N}=\mathcal{F}_{M N}(x) \quad \text { and } \quad \mathcal{F}_{a M}=\mathcal{F}_{a b}=0 .
\end{aligned}
$$

Here $g_{a b}(y)$ is an $n$-dimensional maximally-symmetric metric, whose curvature scalar is: $g^{a b} R_{a b}=n(n-1) K$, for constant $K$. Furthermore, the above configuration is the most general one consistent with this maximal symmetry, which ensures that solutions to the truncated action are guaranteed also to be solutions of the full higher-dimensional equations. (Such a configuration is called a 'consistent' truncation [26].)

With this ansatz the action of the truncated 6D theory becomes [23, 24]

$$
\begin{aligned}
S=\frac{1}{\kappa^{2}} \int \mathrm{d}^{6} x \sqrt{-g}\left[R-\frac{n(n+4)}{4} \partial_{M} \varphi \partial^{M} \varphi-\frac{\kappa^{2}}{4} e^{n \varphi / 2} \operatorname{Tr} F^{2}\right. & \\
& \left.-\Lambda e^{-n \varphi / 2}+K n(n-1) e^{-(n+4) \varphi / 2}\right],
\end{aligned}
$$

where we define $\kappa^{2}:=\kappa_{D}^{2} / V$ and $F_{M N}:=V^{1 / 2} \mathcal{F}_{M N}$, with $V$ the volume of the $n$-dimensional manifold computed with the metric $g_{a b}$. Finally, defining

$$
\kappa \sigma=\sqrt{n(n+4)} \varphi \quad \text { and } \quad \zeta^{2}=\frac{n}{n+4}
$$

and so $n=4 \zeta^{2} /\left(1-\zeta^{2}\right)$, allows the action to be written

$$
\begin{aligned}
S=\frac{1}{\kappa^{2}} \int \mathrm{d}^{6} x \sqrt{-g}\left[R-\frac{\kappa^{2}}{4} \partial_{M} \sigma \partial^{M} \sigma\right. & -\frac{\kappa^{2}}{4} e^{\zeta \kappa \sigma / 2} \operatorname{Tr} F^{2} \\
& \left.-\Lambda e^{-\zeta \kappa \sigma / 2}+K \frac{4 \zeta^{2}\left(5 \zeta^{2}-1\right)}{1-\zeta^{2}} e^{-\kappa \sigma / 2 \zeta}\right] .
\end{aligned}
$$

This shows that the $6 \mathrm{D}$ supergravity action, eq. (2.2), is obtained in the formal limit where $K=0$ and $\zeta \rightarrow 1$ (and so $n \rightarrow \infty$ ), provided we also identify $\Lambda=8 g_{1}^{2} / \kappa^{2}$.

\subsubsection{The Rugby Ball and its Decay}

As an application consider the following simple monopole-supported compactification from $D$ to $D-2$ dimensions:

$$
\begin{aligned}
\mathrm{d} s_{D}^{2} & =g_{A B} \mathrm{~d} x^{A} \mathrm{~d} x^{B}+a^{2}\left(\mathrm{~d} \theta^{2}+\sin ^{2} \theta \mathrm{d} \phi^{2}\right) \\
\mathcal{F}_{\theta \phi}^{a} & =\frac{q_{D}^{a}}{2} \sin \theta
\end{aligned}
$$

where $A, B, . .=0,1, \ldots, n+3$, for which directions the $d=(D-2)=(4+n)$-dimensional metric is

$$
R_{A B}=(d-1) \lambda_{d} g_{A B}=(D-3) \lambda_{d} g_{A B} .
$$

\footnotetext{
${ }^{17}$ The indices $\Gamma, \Lambda, .$. run from 0 to $n+5$, while indices $a, b, .$. run from 1 to $n$ and $6 \mathrm{D}$ indices $M, N, .$. run from 0 to 5 as before. We reserve $A, B,$. to run from 0 to $n+3$ in later applications.
} 
Using this ansatz in the $D$-dimensional equations of motion allows their content to be boiled down to

$$
\begin{aligned}
(D-3) \lambda_{d} & =-\frac{1}{D-2}\left[\frac{\kappa_{D}^{2} q_{D}^{2}}{8 a^{4}}-\Lambda\right] \\
\frac{1}{a^{2}} & =\frac{\kappa_{D}^{2} q_{D}^{2}}{8 a^{4}}+(D-3) \lambda_{d}
\end{aligned}
$$

whose solutions are

$$
\begin{aligned}
& a_{ \pm}^{2}=\frac{(D-2)}{2 \Lambda}\left[1 \pm \sqrt{1-\frac{(D-3)}{2(D-2)^{2}} \Lambda \kappa_{D}^{2} q_{D}^{2}}\right] \\
& \lambda_{d}=\frac{1}{(D-3)^{2}}\left[\Lambda-\frac{1}{a_{ \pm}^{2}}\right] .
\end{aligned}
$$

Eliminating $q_{D}^{2}$ gives the 2 -sphere radius in terms of the $d$-dimensional curvature:

$$
a_{ \pm}^{2}=\frac{1}{\Lambda-(D-3)^{2} \lambda_{d}}=\frac{1}{\Lambda-(n+3)^{2} \lambda_{d}}
$$

Applying these results to an initial geometry $\operatorname{Mink}_{4} \times S_{2}$ supported by an unstable monopole having charge $q^{2}=q_{i}^{2}$ shows that the parameter $\Lambda$ must be tuned to

$$
\Lambda=\frac{1}{a_{i}^{2}}=\frac{8}{\kappa_{D}^{2} q_{D i}^{2}}=\frac{8}{\kappa^{2} q_{i}^{2}},
$$

in which the final equality cancels the factors of extra-dimensional volume, $V$, that appear in the relations between the $D$ - and 6-dimensional versions of $\kappa$ and $q^{2}$. Dimensionally reducing this geometry on $n$ of the flat directions then trivially reproduces the rugby-ball solution, eq. (2.10), of $6 \mathrm{D}$ supergravity (whose decay we wish to study).

As in $\S 3$ we suppose the endpoint of the instability in the $D$-dimensional system also to be given by solutions to these same equations, but for the smaller charge of the final stable monopole: $q_{D f}^{2}<q_{D i}^{2}$. And eq. (4.67) implies that shrinking $q^{2}$ makes $a_{+}^{2}$ get larger while $a_{-}^{2}$ gets smaller, which eq. (4.68) in turn implies $\lambda_{d+}$ is positive while $\lambda_{d-}$ is negative. As in $\S 3$ this predicts the endpoint to be a smaller monopole-supported sphere, with negatively curved large directions.

The idea now is to dimensionally truncate the endpoint monopole-supported $D$-dimensional geometry on $n$ of its AdS dimensions, thereby obtaining a candidate endpoint solution for the 6D EYM-dilaton system. To this end it is useful to rewrite the $D$-dimensional metric in terms of flat spatial slicings

$$
\begin{aligned}
\mathrm{d} s_{D}^{2}= & {\left[\mathrm{d} x^{2}+e^{2 \sqrt{-\lambda_{d}} x}\left(-\mathrm{d} t^{2}+\delta_{i j} \mathrm{~d} x^{i} \mathrm{~d} x^{j}+\delta_{a b} \mathrm{~d} y^{a} \mathrm{~d} y^{b}\right)\right]+a_{-}^{2} \mathrm{~d} \Omega_{2}^{2} } \\
= & e^{-n \sqrt{-\lambda_{d}} x / 2}\left[-e^{(2+n / 2) \sqrt{-\lambda_{d}} x} \mathrm{~d} t^{2}+e^{n \sqrt{-\lambda_{d}} x / 2} \mathrm{~d} x^{2}+e^{(2+n / 2) \sqrt{-\lambda_{d}} x} \delta_{i j} \mathrm{~d} x^{i} \mathrm{~d} x^{j}\right. \\
& \left.+a_{-}^{2} e^{n \sqrt{-\lambda_{d}} x / 2} \mathrm{~d} \Omega_{2}^{2}\right]+e^{2 \sqrt{-\lambda_{d}} x} \delta_{a b} \mathrm{~d} y^{a} \mathrm{~d} y^{b},
\end{aligned}
$$


where $i, j, .$. run from 1 to 2 , while (as before) $a, b, .$. run from 4 to $4+n$, and $\mathrm{d} \Omega_{2}^{2}$ denotes the standard metric on the unit 2-sphere.

Comparing this last expression with the ansatz, eq. (4.60) allows the dilaton to be read off from the $x$-dependence of the $n$-dimensional truncated metric, giving $\varphi=\sqrt{-\lambda_{d}} x$, or

$$
\kappa \sigma=\frac{4 \zeta}{1-\zeta^{2}} \sqrt{-\lambda_{d}} x
$$

Using this in eq. (4.60) then also allows the $6 \mathrm{D}$ metric to be identified. Making the change of variables

$$
z=\int e^{n \sqrt{-\lambda_{d}} x / 4} \mathrm{~d} x=\frac{4}{n \sqrt{-\lambda_{d}}} e^{n \sqrt{-\lambda_{d}} x / 4}
$$

allows the truncated $6 \mathrm{D}$ solution to be written

$$
\mathrm{d} s^{2}=-\left(\frac{z}{L_{n}}\right)^{2+8 / n} \mathrm{~d} t^{2}+\mathrm{d} z^{2}+\left(\frac{z}{L_{n}}\right)^{2+8 / n} \delta_{i j} \mathrm{~d} x^{i} \mathrm{~d} x^{j}+\left(\frac{z}{L_{n}}\right)^{2} a_{-}^{2} d \Omega_{2}^{2},
$$

and

$$
\kappa \sigma=\frac{4}{\zeta} \ln \left(\frac{z}{L_{n}}\right)
$$

where the length scale $L_{n}$ is defined by

$$
\frac{1}{L_{n}}:=\frac{n \sqrt{-\lambda_{d}}}{4}=\frac{n}{4(n+3)} \sqrt{\frac{1}{a_{-}^{2}}-\frac{1}{a_{i}^{2}}},
$$

and the expression for $\lambda_{d}$ in terms of $a_{-}$and $a_{i}$ is used. The final step is to take $n \rightarrow \infty$ $(\zeta \rightarrow 1)$ to recover $6 \mathrm{D}$ Nishino-Sezgin supergravity. Both $L$ and $a_{-}$remain finite in this limit, with

$$
\frac{1}{L}=\lim _{n \rightarrow \infty} \frac{1}{L_{n}}=\frac{1}{4} \sqrt{\frac{1}{a_{-}^{2}}-\frac{1}{a_{i}^{2}}}
$$

and

$$
\lim _{n \rightarrow \infty} a_{-}^{2}=\lim _{n \rightarrow \infty} \frac{(n+4)}{2 \Lambda}\left[1 \pm \sqrt{1-\frac{(n+3)}{2(n+4)^{2}} \Lambda \kappa^{2} q_{f}^{2}}\right]=\frac{\kappa^{2} q_{f}^{2}}{8} .
$$

Combining all expression gives the final result for the candidate endpoint solution to gauged chiral 6D supergravity

$$
\begin{aligned}
& \mathrm{d} s^{2}=\mathrm{d} z^{2}+\left(\frac{z}{L}\right)^{2}\left[-\mathrm{d} t^{2}+\delta_{i j} \mathrm{~d} x^{i} \mathrm{~d} x^{j}\right]+\left(\frac{z}{L}\right)^{2} a_{-}^{2} \mathrm{~d} \Omega_{2}^{2}, \\
& \kappa \sigma=4 \ln \left(\frac{z}{L}\right) \quad \text { and } \quad F_{\theta \phi}^{a}=\frac{q_{f}^{a}}{2} \sin \theta .
\end{aligned}
$$

One can check directly that this configuration indeed solves the 6D supergravity equations, and in fact can be recognized as one of the scaling solutions found in [8], but with the scaling occurring along a 4D spatial coordinate, z, rather than time. Also noteworthy is the relation 
this solution implies between the sphere's radius, $r$, and the dilaton: $r^{2}=e^{\kappa \sigma / 2} a_{-}^{2}$, which is also familiar (but $z$-independent) from the Salam-Sezgin compactification [1].

The solution eventually breaks down for small $z$ due to the singularity as $z \rightarrow 0$, where both the dilaton and the 6D Ricci scalar,

$$
R=\frac{2\left(L^{2}-10 a_{-}^{2}\right)}{z^{2} a_{-}^{2}},
$$

blow up. Since this singularity has no counterpart in the higher dimensional $\mathrm{AdS}_{4+n} \times S_{2}$ EYM solution, its emergence is a consequence of taking the limit $n \rightarrow \infty$. The finite- $n$ geometries may be regarded in this way as providing resolutions of this singularity, along the lines of the higher-dimensional resolution of dilatonic black hole singularities in string theory described in ref. [27].

At large $z$ the radius of the compact 2-sphere becomes very large, implying an eventual breakdown of the $4 \mathrm{D}$ effective theory even at very low energies. It is instructive to ask how the metric varies in the 4D Einstein frame, especially since the dependence on $z$ only arises as an overall conformal factor (as may be seen using the coordinate change $u=\ln (z / L)$ ),

$$
\mathrm{d} s^{2}=e^{2 u}\left(\eta_{\mu \nu} \mathrm{d} x^{\mu} \mathrm{d} x^{\nu}+a_{-}^{2} \mathrm{~d} \Omega_{2}^{2}\right)
$$

implying the breaking of the 4D maximal symmetry therefore drops out of conformally invariant quantities. Since the volume of the 2 compact dimensions varies as $V_{2}=(z / L)^{2} a_{-}^{2} \propto e^{2 u}$, the 4D Einstein frame metric scales with $u$ as $g_{\mu \nu}^{(E)}=e^{2 u} g_{\mu \nu}$, which is again $u$-dependent, and in fact turns out to be the same geometry as that of the 6D Einstein frame.

\section{Stability}

The stability of this solution follows from that of the corresponding oxidised solution, $\mathrm{AdS}_{4+n} \times S_{2}$, since the fluctuations in the $6 \mathrm{D}$ model are a sub-sector of those in the oxidised model, allowing us to conclude that our proposed endpoint is a stable solution, without performing the linearlised stability analysis from scratch. Fluctuations in the $(6+n) \mathrm{D}$ EYM model divide into two decoupled sectors:

1. The metric fluctuations and gauge field fluctuations in the direction of the $U(1)$ monopole in the Lie Algebra. These were studied in [28], where they were found to be stable, in the sense that none violate the Breitenlohner-Freedman bound.

2. The gauge field fluctuations orthogonal to the $U(1)$ monopole. We argued above that the presence of instabilities in this sector for $\mathrm{Mink}_{4} \times S_{2}$ [6, 2] generalise to higher dimensions and so these modes are also unstable in the $\operatorname{Mink}_{4+n} \times S_{2}$ theory. The identical argument shows that stable monopoles in $\operatorname{Mink}_{4} \times S_{2}$ oxidise to configurations that are also stable in $\operatorname{Mink}_{4+n} \times S_{2}$. The same conclusion should also apply for $\mathrm{AdS}_{4+n} \times S_{2}$, since the Kaluza-Klein mass operator does not depend on the curvature of the external geometry, but only on the curvature of the internal geometry and the internal flux. 


\section{Energy}

The higher-dimensional picture also argues for there being an energetic criterion which favours these new solutions as having smaller energy then the initial, unstable one. Given the non-trivial profile for the dilaton in the large dimensions, an appropriate definition for the energy is the sum of the 4D dilatonic gradient energy and the potential energy of the 4D effective theory defined in (4.51), which emerges from the gradient, magnetic and potential energy in the extra dimensions. Integrating out the extra dimensions, the total energy density (per unit 3D volume) in the Einstein frame, $g_{\mu \nu}^{(E)}=e^{\kappa \sigma / 2} g_{\mu \nu}$, is:

$$
\mathcal{E}_{\text {TOT }}=\frac{e^{-\kappa \sigma}}{\kappa^{2}} \int \mathrm{d}^{2} x \sqrt{g_{2}}\left[\frac{\kappa^{2}}{4} e^{\kappa \sigma / 2} \partial_{z} \sigma \partial^{z} \sigma-R_{(2)}+\frac{\kappa^{2}}{4} \operatorname{Tr} F_{m n} F^{m n}+\Lambda e^{-\kappa \sigma / 2}\right]
$$

where the overall factor of $e^{-\kappa \sigma}$ comes from the Weyl rescaling to the Einstein frame of the $4 \mathrm{D}$ volume factor. A non-trivial gradient energy in the dilaton always gives a positive contribution to the total energy, whereas the 4D potential energy in terms of the dilaton and volume breathing modes is:

$$
\mathcal{E}=-\frac{4 \pi a_{-}^{2}}{\kappa^{2}} e^{-\kappa \sigma} \frac{1}{2 a_{-}^{2}}\left(1-\frac{a_{-}^{2}}{a_{i}^{2}}\right)
$$

Plugging the endpoint configuration (4.79) into (4.82) shows that the potential energy is negative, $-\frac{4 \pi a_{-}^{2}}{\kappa^{2}} \frac{8}{L^{2} z^{4}}$, and beats the gradient energy, $\frac{4 \pi a_{-}^{2}}{\kappa^{2}} \frac{4}{L^{2} z^{4}}$, giving in total:

$$
\mathcal{E}_{T O T}=-\frac{4 \pi a_{-}^{2}}{\kappa^{2}} \frac{4}{L^{2} z^{4}}
$$

This result should be compared to the initial total energy, for which both the $4 \mathrm{D}$ gradient and potential contributions are zero, and so the energy has been lowered.

\subsubsection{The Decay of Warped Configurations}

As a second example we extend the above analysis from sphere-monopole compactifications to the more generic presence of warping, as is required if the two brane tensions are unequal. We know that configurations with monopole numbers $\left|N_{I}\right| \geq 2$ are also unstable in warped brane-world compactifications with positive-tension brane sources [2]. We now seek the endpoint of this stability, as indicated by the above oxidation/reduction technique.

To do so we first display a warped solution to the $(n+6)$-dimensional EYM system with cosmological constant, which reduces to the warped Minkowski solution of the 6D EYM $\sigma$ theory. As previously, the instability of the 6D solution is shared by its higher dimensional representation 18 We identify a plausible endpoint in the higher-dimensional EYM theory, and reduce it to identify the corresponding candidate endpoint in 6D supergravity.

\footnotetext{
${ }^{18}$ Note, however, that the direct linearized analyis made in 2] does not extend to warped solutions in $(6+n) D$ because the bilinear action for the modes of relevance depends on $(n+4)$ in the warped case [25].
} 


\section{The higher-dimensional warped solution}

We again start from the $(n+6)$ dimensional EYM action (4.57)

$$
S=\frac{1}{\kappa_{D}^{2}} \int \mathrm{d}^{D} x \sqrt{-g_{D}}\left[R_{(D)}-\frac{\kappa_{D}^{2}}{4} \operatorname{Tr} \mathcal{F}^{2}-\Lambda\right] .
$$

The relevant static warped solution to the corresponding field equations is obtained by a Weyl rotation of a known black-hole like solution [29], as was done in [15] (a similar solution and method were also used in [30]). The result is

$$
\begin{aligned}
\mathrm{d} s_{D}^{2} & =r^{2} g_{A B} \mathrm{~d} x^{A} \mathrm{~d} x^{B}+\frac{\mathrm{d} r^{2}}{h_{D}(r)}+\epsilon_{D}^{2} h_{D}(r) d \phi^{2} \\
\text { and } \quad \mathcal{F}_{r \phi} & =-\frac{\epsilon_{D} q_{D}^{a} Q^{a}}{r^{n+4}},
\end{aligned}
$$

where $g_{A B}$ is a $d=(n+4)$-dimensional, maximally symmetric metric, with $R_{A B}=(d-$ 1) $\lambda_{d} g_{A B}$, and we have introduced an additional parameter, $\epsilon_{D}$, which will allow us to reach the unwarped compactifications via a smooth limit. The function $h_{D}(r)$ is given explicitly by

$$
h_{D}(r)=\lambda_{d}+\frac{M}{r^{n+3}}-\frac{\Lambda r^{2}}{(n+4)(n+5)}-\frac{\kappa_{D}^{2} q_{D}^{2}}{2(n+4)(n+3) r^{2(n+3)}},
$$

where $M$ is an integration constant that can be positive or negative. This solution can also be found by solving directly the equations of motion, for which the Einstein equations reduce under the above ansatz to

$$
\begin{aligned}
& R_{A B}=-g_{A B}\left[-\frac{(n+3) \lambda_{d}}{r^{2}}+\frac{h_{D}^{\prime}}{r}+\frac{(n+3) h_{D}}{r^{2}}\right]=-g_{A B}\left[\frac{\kappa_{D}^{2} q_{D}^{2}}{8 r^{2(n+4)}}-\frac{\Lambda}{4}\right] \\
& R_{m n}=-\frac{1}{2} g_{m n}\left[h_{D}^{\prime \prime}+\frac{(n+4) h_{D}^{\prime}}{r}\right]=g_{m n}\left[\frac{3 \kappa_{D}^{2} q_{D}^{2}}{8 r^{2(n+4)}}+\frac{\Lambda}{4}\right] .
\end{aligned}
$$

Ref. [30] shows that this yields the desired warped 6D solutions to Nishino-Sezgin supergravity found in [15] once the limit $n \rightarrow \infty$ is taken, making them a good starting point for seeking the endpoint of the decay of the underlying monopole.

The solution above (4.86, 4.88) is very similar to that studied in [15], Section 3 (see also [14, 16]). The geometry is well defined in the region where the metric function $h_{D}(r)$ is positive, and this implies $M>0$ when $\lambda_{d} \leq 0$, while $M$ can be negative for $\lambda_{d}>0$. Similar to what is shown in [15], the geometry pinches off at the points where $h_{D}(r)$ vanishes. There are two such real roots, $r_{ \pm}$, since $h_{D}(r) \rightarrow-\infty$ as $r \rightarrow 0$ and $r \rightarrow \infty$, and changes sign only twice.

Moreover, because $h_{D}$ vanishes linearly near $r=r_{ \pm}$, being well approximated by $h_{D}(r) \sim$ $h_{D}^{\prime}\left(r_{ \pm}\right)\left(r-r_{ \pm}\right)$, the 2D internal metric is approximately conical at these points, with:

$$
\mathrm{d} s_{2}^{2} \sim \mathrm{d} R_{ \pm}^{2}+\left(1-\frac{\delta_{ \pm}}{2 \pi}\right)^{2} R_{ \pm}^{2} \mathrm{~d} \phi^{2}
$$


Here $R_{ \pm}=2 \sqrt{\left(r-r_{ \pm}\right) / h_{D}^{\prime}\left(r_{ \pm}\right)}$, and the deficit angles are given by:

$$
\frac{\delta_{ \pm}}{2 \pi}=1-\frac{1}{2}\left|\epsilon_{D} h_{D}^{\prime}\left(r_{ \pm}\right)\right|
$$

These singularities are sourced by codimension-two branes, with actions

$$
S_{\text {brane }}=-\mathcal{T}_{ \pm} \int d^{D-2} y \sqrt{-\gamma_{ \pm}}
$$

and whose tensions satisfy $\kappa_{D}^{2} \mathcal{T}_{ \pm}=2 \delta_{ \pm}$. On reduction to the $6 \mathrm{D}$ theory these become 3-branes with tensions $T_{ \pm}$given by $\mathcal{T}_{ \pm} V$.

Finally, since the internal space is compact, there is as usual a Dirac quantization condition for the magnetic flux. Covering the space with two patches that respectively incorporate $r_{ \pm}$, à la $\mathrm{Wu}$ and Yang, allows the gauge potential to be written

$$
\mathcal{A}_{ \pm}=\frac{\epsilon_{D} q_{D}^{a} Q_{a}}{(n+3)}\left(\frac{1}{r^{n+3}}-\frac{1}{r_{ \pm}^{n+3}}\right) \mathrm{d} \phi
$$

These are related by a single-valued gauge transformation on the overlap only if

$$
-g e_{a I} \frac{\epsilon_{D} q_{D}^{a}}{n+3}\left(\frac{1}{r_{+}^{n+3}}-\frac{1}{r_{-}^{n+3}}\right)=N_{I}
$$

where $e_{a I}$ are the adjoint charges discussed in earlier sections, and $N_{I}$ is an integer.

In order to have an expression for $h$ in terms of the two real roots, we can write it as follows $(\ell:=n+3)$ :

$$
\begin{aligned}
h_{D}(r)= & \lambda_{d}\left[1-\frac{r_{+}^{\ell}}{r^{\ell}}\right]\left[1-\frac{r_{-}^{\ell}}{r^{\ell}}\right] \\
& +\frac{\Lambda}{(\ell+1)(\ell+2)} \frac{1}{r_{+}^{2}\left(r_{+}^{\ell}-r_{-}^{\ell}\right)} \frac{1}{r^{2 \ell}}\left[r^{\ell}\left(r_{+}^{2 \ell+2}-r_{-}^{2 \ell+2}\right)-r^{2 \ell+2}\left(r_{+}^{\ell}-r_{-}^{\ell}\right)\right.
\end{aligned}
$$

where now it is clear that $h\left(r_{ \pm}\right)=0$. By comparing (4.88 and 4.96), the parameters $r_{ \pm}$can be related to the original parameters of the solution as:

$$
\begin{aligned}
M & =\frac{\Lambda}{(\ell+1)(\ell+2)} \frac{\left(r_{+}^{2(\ell+1)}-r_{-}^{2(\ell+1)}\right)}{r_{+}^{2}\left(r_{+}^{\ell}-r_{-}^{\ell}\right)}-\lambda_{d}\left(r_{+}^{\ell}+r_{-}^{\ell}\right) \\
\frac{\kappa_{D}^{2} q_{D}^{2}}{2 \ell(\ell+1)} & =\left(r_{+} r_{-}\right)^{\ell}\left[\frac{\Lambda}{(\ell+1)(\ell+2)} \frac{\left(r_{+}^{\ell+2}-r_{-}^{\ell+2}\right)}{r_{+}^{2}\left(r_{+}^{\ell}-r_{-}^{\ell}\right)}-\lambda_{d}\right]
\end{aligned}
$$

and moreover $r_{+}=1$, which amounts to a choice of coordinates. Meanwhile, the tensions of 
the branes can be related to the bulk parameters via Eq. (4.92):

$$
\begin{aligned}
& 1-\frac{\mathcal{T}_{+} \kappa_{D}^{2}}{4 \pi}= \\
& \frac{1}{2}\left|\frac{\epsilon_{D}}{r_{-}^{\ell}-1}\left(\left(\ell+2-2(\ell+1) r_{-}^{\ell}+\ell r_{-}^{2 \ell+2}\right) \frac{\Lambda}{(\ell+1)(\ell+2)}-\ell\left(r_{-}^{\ell}-1\right)^{2} \lambda\right)\right| \\
& 1-\frac{\mathcal{T}_{-} \kappa_{D}^{2}}{4 \pi}= \\
& \frac{1}{2}\left|\frac{\epsilon_{D}}{r_{-}^{\ell+1}\left(r_{-}^{\ell}-1\right)}\left(\left(-\ell+2(\ell+1) r_{-}^{\ell+2}-(\ell+2) r_{-}^{2 \ell+2}\right) \frac{\Lambda}{(\ell+1)(\ell+2)}+\ell\left(r_{-}^{\ell}-1\right)^{2} \lambda\right)\right| .
\end{aligned}
$$

\section{Unwarped limit}

As an aside, we show how the above higher dimensional warped background reduces to the known unwarped solution as the warp factor goes to one, $r_{-} \rightarrow r_{+}$. This limit can be taken by making the change of coordinates:

$$
r=\frac{r_{+}}{2}((1+\alpha)+(1-\alpha) \cos \theta)
$$

where we have defined $\alpha:=r_{-} / r_{+}$. We then take $\alpha=1+\xi$ together with the limit $\xi \rightarrow 0$, but insist that $\xi \epsilon_{D} \rightarrow \varepsilon_{D}$, a finite constant. In this way, the metric assumes the form of the rugbyball

$$
d s_{D}^{2}=g_{A B} d x^{A} d x^{B}+a^{2}\left(d \theta^{2}+\beta^{2} \sin ^{2} \theta d \phi^{2}\right)
$$

where the radius and deficit angle are, respectively,

$$
\begin{aligned}
a^{2} & =\frac{1}{\Lambda-\ell^{2} \lambda} \quad \text { and } \\
\beta^{2} & =\frac{\varepsilon_{D}^{2}}{4 a^{4}},
\end{aligned}
$$

and the gauge field is that of the monopole

$$
\mathcal{A}_{ \pm}=\frac{\varepsilon_{D} q_{D}^{a} Q_{a}}{2}(\cos \theta \mp 1) \mathrm{d} \phi .
$$

The quantisation condition (4.95) reduces to $-g \varepsilon_{D} q_{D}^{a} e_{a I}=N_{I}$, and Eq. (4.98) tells us that:

$$
\ell \lambda=\frac{1}{(\ell+1)}\left[\Lambda-\frac{\kappa_{D}^{2} \varepsilon_{D}^{2} q_{D}^{2}}{8 \beta^{2} a^{4}}\right],
$$

which is precisely one of the constraints encountered from the equations of motion for the unwarped rugbyball. Meanwhile, the boundary conditions (4.99, 4.100) also reduce to the expected ones:

$$
\begin{aligned}
& \frac{\mathcal{T}_{+} \kappa_{D}^{2}}{4 \pi}=1-\beta \\
& \frac{\mathcal{T}_{-} \kappa_{D}^{2}}{4 \pi}=1-\beta .
\end{aligned}
$$


We obtain an additional constraint by putting together Eqs. (4.98) and (4.99, 4.100): after some manipulation one arrives at the condition $\lambda=0$. Therefore, we are able to take the unwarped limit only for flat $(n+4) \mathrm{D}$ slices, and the warped generalizations for the $\mathrm{dS}_{4+n} \times S_{2}$ and $\mathrm{AdS}_{4+n} \times S_{2}$ solutions are yet to be discovered. Finally, taking furthermore $\beta \rightarrow 1$ we recover the equations for the sphere (4.66, 4.69).

\section{The Warped Endpoints: Upstairs and Downstairs}

Back to the main line of argument, having established the higher dimensional warped configurations that are assumed in the presence of 3-branes, we now ask what happens to these configurations when they are unstable. As described above, we expect both the 6D and $D$ dimensional warped solutions to be unstable when there are monopoles numbers $\left|N_{I}\right|>1$. The monopole numbers depend on the charges present, as well as the parameters $q_{D}^{a}, \epsilon_{D}$ and $r_{-}$, via the Dirac Quantisation condition (4.95). The $d=4+n$-dimensional curvature, $\lambda_{d}$, completes the description of the solution (4.86 4.88), but not all of these parameters are independent, due to the equations of motion (4.98, 4.99, 4.100). Thus we can specify a given solution completely with one parameter, say, $q_{D}^{a}$. Beginning with an unstable solution, $q_{D i}^{a}$, the monopole field strength will decay conserving its topological flux, as we have seen previously, and the geometry will adjust appropriately according to the equations of motion. A reasonable endpoint in the $D$-dimensional EYM theory is then a warped configuration within the same class (4.86, 4.88), with new parameters $q_{D f}^{a}, \epsilon_{D f}, r_{-_{f}}$ and $\lambda_{d_{f}}$.

It remains to play the same game as in unwarped case to discover how the geometry and dilaton respond to the decay of the monopole in $6 \mathrm{D}$ supergravity. The rules of the game are by now familiar; we begin with a warped dilatonic $6 \mathrm{D}$ model, tuning the bulk cosmological constant, $\Lambda$, to allow for flat 4D slices in the initial unstable configuration, with monopole strength $q^{a}=q_{i}^{a}$ and brane tensions $T_{ \pm}$. Uplifting this model to a nondilatonic D-dimensional theory, it is easy to see that the decay of the monopole to its stable topological cousin curves the $(4+n) \mathrm{D}$ slices from $\lambda_{d_{i}}=0$ to $\lambda_{d_{f}} \neq 0$. Now we dimensionally reduce the stable D-dimensional solution, and take the $n \rightarrow \infty$ limit, in order to recover the geometry and dilaton profile in the 6D supergravity model.

The dimensional reduction is performed as in the previous section. To allow a well-defined $n \rightarrow \infty$ limit, we further make the change of coordinates $\rho=r^{2+\frac{n}{2}}$, along with the parameter

redefinitions $\rho_{-}=r_{-}^{2+\frac{n}{2}}, \epsilon=2 \epsilon_{D} / n$ and $\lambda=n^{2} \lambda_{d}$. Moreover, we define the function $h(\rho)$ as $h(\rho)=\lim _{n \rightarrow \infty} \frac{n^{2} \rho}{4} h_{n}(\rho):$

$$
h(\rho)=(\lambda-\Lambda) \frac{\rho}{4}\left[1-\frac{1}{\rho^{2}}\right]\left[1-\frac{\rho_{-}^{2}}{\rho^{2}}\right] .
$$

Finally, the solution to the 6D supergravity can be written:

$$
\begin{gathered}
d s_{6}^{2}=\rho d z^{2}+\rho\left(\frac{z}{L}\right)^{2}\left(-d t^{2}+\delta_{i j} d x^{i} d x^{j}\right)+\left(\frac{z}{L}\right)^{2}\left(\frac{d \rho^{2}}{h(\rho)}+\epsilon^{2} h(\rho) d \phi^{2}\right) \\
\kappa \sigma=4 \ln \left(\frac{z}{L}\right)+2 \ln \rho \quad F_{\rho \phi}=-\frac{\epsilon q^{a}}{\rho^{3}} Q_{a}
\end{gathered}
$$


where, assuming $\lambda<0$, we have defined $z$ as in Eq. (4.73), $4 / L=\sqrt{-\lambda}$ and the quantization condition takes the familiar form:

$$
-g e_{a I} \frac{\epsilon q^{a}}{2}\left(1-\frac{1}{\rho_{-}^{2}}\right)=N_{I}
$$

where we recall the choice of coordinates such that $\rho_{+}=1$. The parameters describing the background, $\lambda, \epsilon$ and $\rho_{-}$, are given as above in terms of $q_{f}, T_{ \pm}$, using (4.98]4.99]4.100) in the limit $n \rightarrow \infty$ :

$$
\begin{aligned}
\lambda & =\Lambda-\frac{\kappa^{2} q_{f}^{2}}{2 \rho_{-}^{2}} \\
\left(1-\frac{T_{+} \kappa^{2}}{4 \pi}\right)^{2} & =\left(\frac{\epsilon}{4} \frac{1}{\left(\rho_{-}^{2}-1\right)}\left(\left(1-2 \rho_{-}^{2}+\rho_{-}^{4}\right) \Lambda-\left(\rho_{-}^{2}-1\right)^{2} \lambda\right)\right)^{2} \\
\left(1-\frac{T_{-} \kappa^{2}}{4 \pi}\right)^{2} & =\left(\frac{\epsilon}{4} \frac{1}{\rho_{-}^{2}\left(\rho_{-}^{2}-1\right)}\left(\left(-1+2 \rho_{-}^{2}-\rho_{-}^{4}\right) \Lambda+\left(\rho_{-}^{2}-1\right)^{2} \lambda\right)\right)^{2} .
\end{aligned}
$$

It is a simple exercise to invert these expressions. Then, the initial tuning of $\Lambda$ gives:

$$
\Lambda=\frac{\kappa^{2} q_{i}^{2}}{2} \frac{\left(1-\frac{T_{+} \kappa^{2}}{4 \pi}\right)}{\left(1-\frac{T_{-} \kappa^{2}}{4 \pi}\right)}
$$

whereas the final solution parameters are:

$$
\begin{aligned}
\lambda & =\Lambda-\frac{\kappa^{2} q_{f}^{2}}{2} \frac{\left(1-\frac{T_{+} \kappa^{2}}{4 \pi}\right)}{\left(1-\frac{T_{-} \kappa^{2}}{4 \pi}\right)} \\
\epsilon & = \pm \frac{32 \pi}{\kappa^{2} q_{f}^{2}} \frac{\left(1-\frac{T_{+} \kappa^{2}}{4 \pi}\right)^{2}}{\left(T_{+} \kappa^{2}-T_{-} \kappa^{2}\right)} \\
\rho_{-} & =\sqrt{\frac{1-\frac{T_{+} \kappa^{2}}{4 \pi}}{1-\frac{T_{-} \kappa^{2}}{4 \pi}}} .
\end{aligned}
$$

In contrast to the unwarped case with or without branes, here we find a unique physical solution with $\lambda<0$ (for the monopole field strength to decay, the combination $\epsilon q^{a}$ must decrease, which, together with $\epsilon \sim 1 / q^{2}$, implies $q_{f}^{2}>q_{i}^{2}$ ). Otherwise, the endpoint in the presence of warping is a straightforward generalization to the one we found in the previous sections, breaking the 4D maximal symmetry, and it is similarly the analogue of the warped scaling solutions found in [8]. Although to establish the stability of this final solution would now require a systematic analysis of its fluctuations, we argue that due to flux conservation, the monopole has nowhere else to go. 


\section{Energy}

Moreover, we now confirm that the energy of our proposed endpoint solution is less than the zero energy of the initial unstable configuration. The total energy density can be defined as in the unwarped case as a sum of 4D gradient and potential energies, (4.82), but now including the warp factor when we integrate out the extra dimensions. Evaluating on the background solution (4.110), we find, just as for the unwarped case, that the gradient energy is $-1 / 2$ of the potential energy, so that the total energy in the Einstein frame $\left(g_{\mu \nu}^{(E)}=(z / L)^{2} g_{\mu \nu}\right)$ is:

$$
\mathcal{E}_{T O T}=-\frac{4 \pi \epsilon}{\kappa^{2}} \frac{\left(1-\rho_{-}^{2}\right)}{L^{2} z^{4}}
$$

and indeed less than zero.

\section{Conclusions}

Compactifications supported by gauge field fluxes were long ago [6, 7] found to be generically unstable, due to tachyonic modes in the non-Abelian degrees of freedom, but the fate that they meet has remained an open question. In this paper, we have explored a number of possible candidates for the endpoint of this instability.

Topological flux conservation suggests that an unstable monopole field decays to the unique, topologically connected, stable monopole [3, 4, 5, and we have determined how the geometry responds to this decay in various scenarios. In the Einstein-Yang Mills theory, with a cosmological constant, a $\operatorname{Mink}_{d} \times S_{2}$ lowers its potential energy by adjusting to $\operatorname{AdS}_{d} \times S_{2}^{\prime}$. In $6 \mathrm{D}$ supergravity, the dilaton precludes such a simple dynamics, and we have argued that it forces the breaking of the maximal symmetry in the non-compact dimensions. By recasting the dilaton as the volume modulus of $n$ fictitious dimensions in a yet-higher dimensional nondilatonic Einstein-Yang Mills theory [23], we were able to find the corresponding solutions explicitly for both unwarped and warped initial configurations, with and without brane sources. The non-trivial profile of the dilaton in 4D generates a singular, static, Kasnerlike geometry that is conformal to (unwarped or warped) $\mathrm{Mink}_{4} \times S_{2}$, where the radius of the 2-sphere grows with the distance from the singularity. How to interpret the naked timelike singularity to which the instability seems to lead is an important open question; does it signal an inconsistency or does it suggest some new physics beyond any supergravity approximation? One way to resolve the singularity is to pass to the higher dimensional Einstein-Yang Mills theory in $(6+n) \mathrm{D}$, in which case the singularity results from projecting the smooth $\mathrm{AdS}_{4+n} \times S_{2}$ geometry onto six dimensions. Such ideas have been discussed in [27]. Moreover, we have shown that the final configuration is perturbatively stable, and that the decay results in a finite total energy which is lower (counting gradient and potential contributions) than the initial one.

We would like to end with a comment. The instability suffered by Yang-Mills sectors in the background of a monopole is the spherical analogue of the Nielsen-Olesen instability that occurs in flat 4D Yang-Mills theory [31]. In that case, it was proposed that condensation of the tachyonic modes leads to the formation of magnetic flux tubes [32], in a rather beautiful imitiation of the vortex formation in superconductor physics [33]. That such a dynamics might also be possible in the present case is certainly an interesting speculation. 


\section{Acknowledgements}

We would like to thank Hyun-Min Lee and Gianmassimo Tasinato for many helpful conversations and insights about extra-dimensional dynamics. CB is supported in part by funds from the Natural Sciences and Engineering Research Council (NSERC) of Canada, CERN and McMaster University. Research at the Perimeter Institute is supported in part by the Government of Canada through NSERC and by the Province of Ontario through MRI. SLP is supported by the Deutsche Forschungsgemeinschaft (DFG) under the Collaborative Research Center (SFB) 676, by the European Union 6th framework program MRTN-CT-503359 "Quest for Unification". IZ is partially supported by the European Union 6th framework program MRTN-CT-2006-035863 "UniverseNet" and SFB-Transregio 33 "The Dark Universe" by the DFG.

\section{References}

[1] A. Salam and E. Sezgin, Phys. Lett. B 147 (1984) 47.

[2] S. L. Parameswaran, S. Randjbar-Daemi and A. Salvio, JHEP 0801 (2008) 051 arXiv:0706.1893 [hep-th]].

[3] R. A. Brandt and F. Neri, Nucl. Phys. B 161 (1979) 253.

[4] R. A. Brandt and F. Neri, Nucl. Phys. B 186 (1981) 84.

[5] S. R. Coleman, The Magnetic Monopole Fifty Years Later, Erice Subnuclear 1981:21 (QCD161:I65:1981)

[6] S. Randjbar-Daemi, A. Salam and J. A. Strathdee, Phys. Lett. B 124 (1983) 345 [Erratum-ibid. B 144 (1984) 455].

[7] A. N. Schellekens, Spaces," Nucl. Phys. B 248, 706 (1984).

[8] A. J. Tolley, C. P. Burgess, C. de Rham and D. Hoover, New J. Phys. 8 (2006) 324 arXiv:hep-th/0608083.

[9] R. Güven, J.T. Liu, C.N. Pope and E. Sezgin, hep-th/0306201.

[10] A. J. Tolley, C. P. Burgess, D. Hoover and Y. Aghababaie, JHEP 0603 (2006) 091 arXiv:hep-th/0512218.

[11] C. P. Burgess, D. Hoover and G. Tasinato, JHEP 0709 (2007) 124 arXiv:0705.3212 [hep-th]].

[12] C. P. Burgess, C. de Rham, D. Hoover and G. Tasinato, arXiv:0812.3820.

[13] H. Nishino and E. Sezgin, Phys. Lett. B 144 (1984) 187.

[14] G. W. Gibbons, R. Gueven and C. N. Pope, Phys. Lett. B 595 (2004) 498 arXiv:hep-th/0307238. 
[15] Y. Aghababaie et al., JHEP 0309, 037 (2003) arXiv:hep-th/0308064.

[16] C. P. Burgess, F. Quevedo, G. Tasinato and I. Zavala, JHEP 0411 (2004) 069 arXiv:hep-th/0408109.

[17] H. M. Lee, JHEP 0805 (2008) 028 [arXiv:0803.2683 [hep-th]].

[18] Y. Aghababaie, C. P. Burgess, S. L. Parameswaran and F. Quevedo, Nucl. Phys. B 680 (2004) 389 arXiv:hep-th/0304256].

[19] T. T. Wu and C. N. Yang, Phys. Rev. D 12 (1975) 3845.

[20] S. Randjbar-Daemi, A. Salam, E. Sezgin and J. A. Strathdee, Phys. Lett. B 151, 351 (1985).

[21] S. D. Avramis and A. Kehagias, JHEP 0510 (2005) 052 arXiv:hep-th/0508172.

[22] G. W. Gibbons and K. i. Maeda, Fields," Nucl. Phys. B 298 (1988) 741.

[23] T. Kobayashi and T. Tanaka, Phys. Rev. D 69, 064037 (2004) arXiv:hep-th/0311197.

[24] T. Kobayashi and M. Minamitsuji, compactifications," JCAP 0707, 016 (2007) arXiv:0705.3500 [hep-th]]; M. Minamitsuji, arXiv:0801.3080 [hep-th].

[25] S. Randjbar-Daemi and M. Shaposhnikov, Nucl. Phys. B 645, 188 (2002) arXiv:hep-th/0206016.

[26] U. Chattopadhyay and A. Karlhede, Phys. Lett. B 139 (1984) 279;

M. J. Duff, "Kaluza-Klein Konsistency," In the proceedings of the 4th Marcel Grossmann Meeting on General Relativity, Rome, Italy, Jun 17-21, 1985 (QC6:M3:1985).

[27] G. W. Gibbons, G. T. Horowitz and P. K. Townsend, Class. Quant. Grav. 12 (1995) 297 arXiv:hep-th/9410073].

[28] R. Bousso, O. DeWolfe and R. C. Myers, Found. Phys. 33 (2003) 297 arXiv:hep-th/0205080.

[29] C. P. Burgess, C. Núñez, F. Quevedo, G. Tasinato and I. Zavala, JHEP 0308 (2003) 056 [arXiv:hep-th/0305211].

[30] S. Mukohyama, Y. Sendouda, H. Yoshiguchi and S. Kinoshita, JCAP 0507 (2005) 013 arXiv:hep-th/0506050.

[31] N. K. Nielsen and P. Olesen, Nucl. Phys. B 144, 376 (1978); H. B. Nielsen and P. Olesen, Nucl. Phys. B 61, 45 (1973); J. Ambjorn, N. K. Nielsen and P. Olesen, Nucl. Phys. B 152, 75 (1979).

[32] J. Ambjorn and P. Olesen, Nucl. Phys. B 170, 265 (1980).

[33] S. Weinberg, Prog. Theor. Phys. Suppl. 86, 43 (1986). 